\title{
Influence of turbulent horseshoe vortex and associated bed shear stress on sediment transport in front of a cylinder
}

\section{Li, Jinzhao; Qi, Meilan; Fuhrman, David R.; Chen, Qigang}

\section{Published in:}

Experimental Thermal and Fluid Science

Link to article, DOI:

10.1016/j.expthermflusci.2018.05.008

Publication date:

2018

Document Version

Peer reviewed version

Link back to DTU Orbit

Citation (APA):

Li, J., Qi, M., Fuhrman, D. R., \& Chen, Q. (2018). Influence of turbulent horseshoe vortex and associated bed shear stress on sediment transport in front of a cylinder. Experimental Thermal and Fluid Science, 97, 444-457. https://doi.org/10.1016/j.expthermflusci.2018.05.008

\section{General rights}

Copyright and moral rights for the publications made accessible in the public portal are retained by the authors and/or other copyright owners and it is a condition of accessing publications that users recognise and abide by the legal requirements associated with these rights.

- Users may download and print one copy of any publication from the public portal for the purpose of private study or research.

- You may not further distribute the material or use it for any profit-making activity or commercial gain

- You may freely distribute the URL identifying the publication in the public portal 


\section{Accepted Manuscript}

Influence of turbulent horseshoe vortex and associated bed shear stress on sediment transport in front of a cylinder

Jinzhao Li, Meilan Qi, David R. Fuhrman, Qigang Chen

PII:

$$
\text { S0894-1777(18)30891-4 }
$$

DOI:

$$
\text { https://doi.org/10.1016/j.expthermflusci.2018.05.008 }
$$

Reference:

$$
\text { ETF } 9473
$$

To appear in:

$$
\text { Experimental Thermal and Fluid Science }
$$

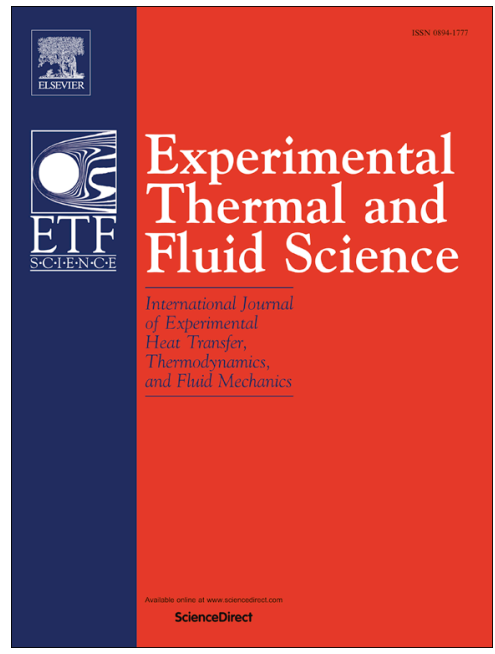

Received Date: 27 December 2017

Revised Date: $\quad 10$ April 2018

Accepted Date: $\quad 11$ May 2018

Please cite this article as: J. Li, M. Qi, D.R. Fuhrman, Q. Chen, Influence of turbulent horseshoe vortex and associated bed shear stress on sediment transport in front of a cylinder, Experimental Thermal and Fluid Science (2018), doi: https://doi.org/10.1016/j.expthermflusci.2018.05.008

This is a PDF file of an unedited manuscript that has been accepted for publication. As a service to our customers we are providing this early version of the manuscript. The manuscript will undergo copyediting, typesetting, and review of the resulting proof before it is published in its final form. Please note that during the production process errors may be discovered which could affect the content, and all legal disclaimers that apply to the journal pertain. 


\section{Influence of turbulent horseshoe vortex and associated bed shear stress on sediment transport in front of a cylinder}

Jinzhao Li ${ }^{\mathrm{a}, \mathrm{d}}$, Meilan $\mathrm{Qi}^{\mathrm{a}, \mathrm{b}^{*}}$, David R. Fuhrman ${ }^{\mathrm{c}}$, Qigang Chen ${ }^{\mathrm{a}}$

${ }^{a}$ Beijing Jiaotong University, School of Civil Engineering, Beijing 100044, China

${ }^{\mathrm{b}}$ Beijing's Key Laboratory of Structural Wind Engineering and Urban Wind Environment, Beijing 100044, China

${ }^{\mathrm{c}}$ Technical University of Denmark, Department of Mechanical Engineering, Section of Fluid Mechanics, Coastal and Maritime Engineering, DK-2800 Kgs. Lyngby, Denmark

${ }^{\mathrm{d}}$ National Engineering Laboratory of Port Hydraulic Construction Technology, Tianjin Research Institute of Water Transport Engineering, Tianjin 300456, China

${ }^{*}$ Corresponding author. E-mail address: mlqi@bjtu.edu.cn (M.L. Qi)

ABSTRACT This study concerns the flow and associated sediment transport in front of a cylinder in steady currents. The study comprises (i) flow characteristics induced by the turbulent horseshoe vortex (THV), (ii) bed shear stress within the THV region, and (iii) predicted sediment transport rates. The velocity fields in front of a wall-mounted circular cylinder were measured using time-resolved particle image velocimetry (PIV). The flow characteristics show that two time-averaged THVs are formed, and the dynamics of instantaneous THVs exhibit a quasi-periodic process from generation to death. Both the mean and fluctuations of bed shear stress within the THV region are significantly amplified, and their values are comparable. The probability density function of the instantaneous bed shear stress exhibits a double-peaked distribution and cannot be represented by the normally-used log-normal distribution for uniform channel-open flows. The comparisons of sediment transport rates where turbulent fluctuations in the bed shear stress are, or are not, taken into account show that the sediment transport rates calculated by the mean bed shear stress are under-predicted. Furthermore, a new sediment transport model incorporating the influence of bed shear stress fluctuations is proposed and validated by comparing the initial scour rate in front of the cylinder.

Keywords: turbulent horseshoe vortex; bed shear stress; sediment transport; particle image velocimetry; cylinder scour 


\begin{tabular}{|c|c|c|c|}
\hline \multicolumn{4}{|c|}{ Nomenclature } \\
\hline$F r$ & Froude number (--) & $v$ & kinematic viscosity $\left(\mathrm{m}^{2} \mathrm{~s}^{-1}\right)$ \\
\hline$R e$ & Reynolds number (--) & $v_{T}$ & turbulent eddy viscosity $\left(\mathrm{m}^{2} \mathrm{~s}^{-1}\right)$ \\
\hline $\operatorname{Re}_{D}$ & cylinder Reynolds number (--) & $\phi$ & dimensionless instantaneous sediment transport rate (--) \\
\hline$I$ & relative bed shear stress fluctuation (--) & $\phi_{m}$ & dimensionless mean sediment transport rate $(--)$ \\
\hline$\tau$ & instantaneous bed shear stress $\left(\mathrm{kg} \mathrm{m}^{-1} \mathrm{~s}^{-2}\right)$ & $r$ & scour rate $\left(\mathrm{m} \mathrm{s}^{-1}\right)$ \\
\hline$\tau_{m}$ & mean bed shear stress $\left(\mathrm{kg} \mathrm{m}^{-1} \mathrm{~s}^{-2}\right)$ & $\rho$ & water density $\left(\mathrm{kg} \mathrm{m}^{-3}\right)$ \\
\hline$\tau_{r m s}$ & RMS of bed shear stress $\left(\mathrm{kg} \mathrm{m}^{-1} \mathrm{~s}^{-2}\right)$ & & \\
\hline
\end{tabular}

\section{Introduction}

When the approach flow encounters a cylinder, the incoming boundary layer separates from the upstream bed due to an adverse pressure gradient imposed by the obstacle, resulting in a so-called turbulent horseshoe vortex (THV) formed in front of the cylinder. The THV is highly relevant to many applications in hydrodynamics and aerodynamics. For example, the THV plays an important role in driving sediment transport and scour around bridge piers in river sand beds, which is a primary reason for bridge failure [1-4]. The flow field around a cylinder becomes complex and interacts strongly with a bottom sand bed, which results in local scour at the cylinder or pier. Therefore, a full understanding of the dynamics of the THV is essential for revealing the underlying mechanisms of sediment transport around piers and preventing unexpected situations in practical applications.

A number of related studies of THV have focused on the time-averaged geometrical characteristics [5-8], the dynamics of THV utilizing particle image velocimetry (PIV) [9-14], eddy-resolving large eddy simulation (LES) [15-17], or detached eddy simulation (DES) [18]. These studies mainly focus on the flow field induced by the THV, however, little literature reports on the relevant influence and relationship of the THV on sediment transport and erosion, which is essential to identify the mechanisms of pier scour and to further improve the prediction accuracy of scour depth. Escauriaza and Sotiropoulos [19] and Link et al. [20] simulated the sediment transport around a cylinder using Lagrangian particle model and found that the interaction of the THV with the wall is the fundamental mechanism for the particle motion and the increase of instantaneous bed shear stress.

Most computations of scour around the cylinder are based on Reynolds-averaged Navier-Stokes (RANS) 
equation models [21-30]. However, RANS modeling results are, by nature, time ensemble averaged, and are incapable of accurately capturing the intense turbulence and detailed vortex structures. For instance, Khosronejad et al. [31] pointed out that RANS models cannot resolve the energetic horseshoe vortex system at the cylinder and bed junction and thus significantly under-predict scour depth in front of the cylinder. Chang et al. [32] also noted that the sediment transport flux calculated by the mean flow field is under-predicted by 2-3 times, when compared with that predicted more correctly by the instantaneous flow fields. Recently, Kim et al. [33, 34] utilized LES modeling that improves the prediction of scour around cylinders, though such LES modeling requires large computational resources, particularly for scour computations, which hinders its application in practice [35].

The bed shear stress, a crucial quantity determining the sediment transport, can be regarded as a key factor bridging the flow characteristics (e.g., THV), sediment transport, and scour. The presence of a THV in front of a cylinder induces an increase of the mean bed shear stress and thus causes sediment transport [36]. Additionally, the instantaneous evolution of a THV directly results in large fluctuations of the instantaneous bed shear stress. Schanderl and Manhart [37] pointed out that the instantaneous bed shear stresses can reach a maximum amplification factor relative to the far field of up to ten in front of a cylinder, indicating the magnitude of these fluctuations. Regarding the influence of bed shear stress fluctuation, Cheng et al. [38] found that the probability density function of the bed shear stress can be described by the log-normal function, which is helpful to establish the relationship between the fluctuating quantity and sediment transport.

Previous investigations imply that the strong turbulence effects (induced by THV) of the flow around a cylinder on sediment transport cannot be neglected when conducting scour computations, which means that the relationship between the turbulent quantities and the sediment transport rate should be established. The results from Cheng et al. [39] show that the sediment transport rate enhanced by turbulence can be expressed as an exponential function of the relative bed shear stress fluctuation. Sumer et al. [40] proposed an empirical equation representing the influence of turbulence on sediment transport based on extensive laboratory experiments. They mainly focus on uniform and unidirectional flows, however, for the rapidly varied flows with reversal vortices in front of a cylinder, little literature reports on the influence of turbulence effects induced by THV on sediment transport.

The objective of this work is to explore the relationship between the THV and associated bed shear stress in front of a circular cylinder, with the primary aim of elucidating the underlying mechanisms 
responsible for sediment transport and scour, as well as establishing the link between the THV-induced bed shear stress fluctuation and sediment transport. We focus on the region influenced by the THV in front of the cylinder. The case of a turbulent flow field in front of a circular cylinder mounted on a flat rigid bed is studied experimentally using the PIV technique. Our experiments do not seek to predict the dynamic deformation of the bed and the development of the scour hole but rather to elucidate the THV-induced fluctuation effect and quantify this effect on sediment transport during the initiation of the scouring process when the bed can be assumed to be flat. As for the later stage of scour development, it is hard to measure the flow field in the scour hole by PIV because the camera view is always blocked by the sediment bed [41]. Alternatively, the flow field in the scour hole is normally experimentally studied by using ADV [7], but this is beyond the scope of this study.

The paper is organized as follows: Section 2 presents details on the experimental setup and flow conditions. In Section 3 we discuss the flow characteristics induced by THV, involving the time-averaged flow field and dynamics of THV. In Section 4 the bed shear stress within the THV region is comprehensively analyzed in terms of its instantaneous variation and statistical quantities, as well as the probability density function of the fluctuations. Section 5 emphasizes the influence of bed shear stress fluctuations on sediment transport by comparing different sediment transport rates, and further proposes a new model of sediment transport rate that incorporates this influence. This new model is also validated by comparing the initial scour rate in front of the cylinder. Finally, conclusions are drawn in Section 6.

\section{Experimental setup}

The experiments were performed in a tilting, water-recirculating, and glass-walled flume, $6 \mathrm{~m}$ long, $0.25 \mathrm{~m}$ wide and $0.25 \mathrm{~m}$ deep, at Bejing Jiaotong University. Flow straightners were used at the entrance to calm the turbulence water, and a tailgate, positioned at the downstream end of the flume, was used to control the water depth. To ensure that the flow becomes fully developed, the measurement section was at $4 \mathrm{~m}$ downstream of the flow entrance by referencing some previous experimental studies [6, 42]. The experimental setup is schematically depicted in Fig. 1. A circular cylinder with diameter $D=4 \mathrm{~cm}$ was used and mounted vertically on the bed in the middle of the test section. The blockage (i.e. flume width to diameter) ratio in the present experiment is 6.25 , which is near the lower limit of the acceptable range indicated by referencing previous relevant studies $[7,13,16]$. The origin of the coordinate system is defined as the center of the cylinder on its bottom surface, with the longitudinal $x$-axis parallel to the flume bed and oriented with the 
main flow, the $y$-axis vertical, and with the $z$-axis normal to the side wall of the flume.

As shown in Fig. 1, the instantaneous, two-dimensional flow fields in the symmetry plane upstream of the cylinder were measured by a PIV system. The reasons that we mainly focus on the symmetry plane are twofold: 1) the scour holes that typically develop are, in fact, reasonably symmetric about the symmetry plane, hence being able to describe the processes along this particular line seems particularly valuable; 2) we used 2D PIV technique in the present work and the time-averaged flow field on the symmetry plane can be regarded as two-dimensional. We attempted to measure the flow field and associated bed shear stress on the other vertical planes off the symmetry, but the obtained two-dimensional results on these planes, such as bed shear stress, are incapable of reasonably predicting the sediment transport due to neglecting the transverse component. In order to perform PIV measurements, the flow was seeded with micro-spheres with mean diameters of $10 \mu \mathrm{m}$ and specific gravity of 1.06 . The flow field of view was illuminated with a $1 \mathrm{~mm}$ thick light sheet generated from a continuous-wave laser. In order to capture the high-resolution particle images, the experiments utilized a high-speed digital CMOS camera with a Nikkor AF $50 \mathrm{~mm}$ f/1.4D lens. The single pulsing mode of PIV is used, and thus the exposure interval of the camera is determined by the frame rate. As the maximum frame rate of the camera is $500 \mathrm{fps}$, to improve the imaging magnification as well as the spatial resolution to capture the detailed structure of vortex, the exposure time between two consecutive frames was set as $2 \mathrm{~ms}$. For each flow case, due to the limited memory of the camera $(1 \mathrm{~GB})$, a sequence of 1000 frames was captured, corresponding to a sampling time of $2 \mathrm{~s}$, and we use 1000 frames to calculate the mean flow field. The solution of instantaneous velocity vectors between two consecutive frames was achieved by using the iterative multigrid image deformation method [43]. The final interrogation window size is $8 \times 8$ pixels which has an overlap of $50 \%$. The size of the camera viewing area is $32 \times 20 \mathrm{~mm}$ such that the spatial resolution is 24.5 pixels $/ \mathrm{mm}$. Based on the flow velocity in our experiment, the interval time of 2 ms assured that the maximum particle displacement between two consecutive frames is 5 pixels, which satisfies the one-quarter rule for PIV correlation analysis [44]. For more specific details of the PIV setup, see the recent work of Qi et al. [45].

Experiments were carried out at two flow conditions, as summarized in Table 1. The Reynolds number $R e$ (which depends on the water depth $h$, the bulk-mean velocity $U_{m}$, and the kinematic viscosity of water $v$ ) is higher than 17,000 to ensure that the incoming open channel flows are fully turbulent. The flow velocity traces also indicate that the flow in these experiments is in the turbulent regime. Two different cylinder Reynolds numbers $\left(R e_{D}=9400\right.$ and 13,500, where $\left.R e_{D}=U_{m} D / v\right)$ are considered. For convenience, each 
case hereafter is named by $\mathrm{C}$ followed by $R e_{D}$, e.g. case C9400 has $R e_{D}=9400$. Experiments with higher $R e_{D}$ were also attempted, but a uniform approaching flow could not be maintained due to the blockage effect of the cylinder. It is worth mentioning that the present value of $R e_{D}$ is relatively small, especially compared with realistic field cases. However, from the viewpoint of numerical modeling (often performed at laboratory scale with small $R e_{D}$ ), it is still valuable to deepen the understanding of mechanisms or for improving scour prediction. For the sake of discussion, the incipient motion conditions (i.e. $U_{m} / u_{c}<1$ or $>1$, where $u_{c}$ is the critical velocity of approach flow for the initiation of sediment motion) have been calculated based on a sediment diameter of $d_{50}=0.6 \mathrm{~mm}$, which will conveniently enable consideration of both clear-water (case C9400) and live-bed (case C13500) scour conditions, respectively. Moreover, this grain size corresponds to that used in forthcoming experimental work involving scour with the same flow condition. In clear-water scour conditions, the upstream flow cannot initiate sediment motion, and sediment transport and scour occur solely by the action of the THV [3]. While in live-bed scour conditions, apart from the effects of the large-scale THV, the small-scale coherent structures from the turbulent boundary layer are also responsible for the near-bed transport [19]. For completeness, both clear-water and live-bed scour conditions are considered in this experiment.

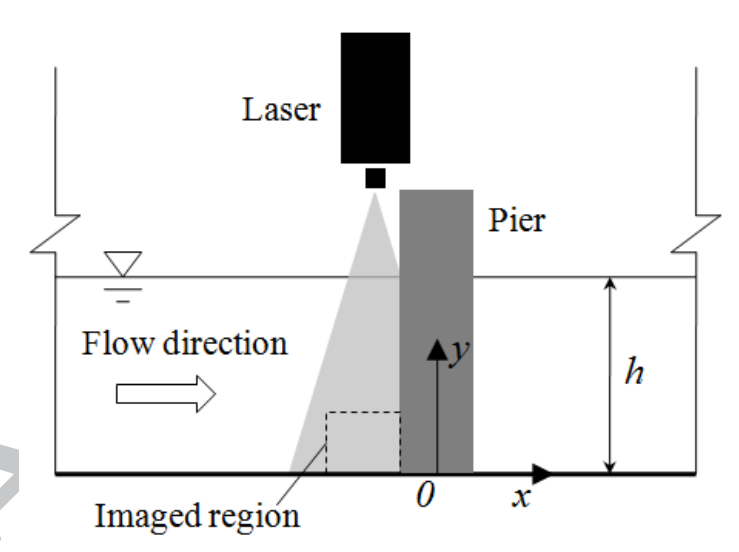

(a)

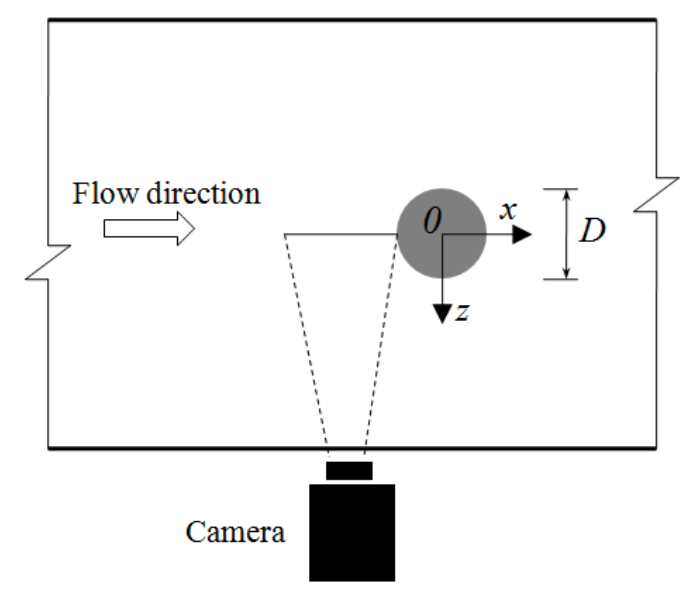

(b)

Figure 1 Schematics of the experimental setup: (a) side view; (b) top view. 
Table 1 Flow conditions: $S$, bed slope; $h$, water depth; $U_{m}$, bulk-mean velocity; $\operatorname{Re}=U_{m} h / \boldsymbol{V}$ ( $\boldsymbol{V}$ is the kinematic viscosity), Reynolds number; $\operatorname{Re} e_{D}=U_{m} D / v$, cylinder Reynolds number; $F r=U_{m} / \sqrt{g h}$, Froude number; $u_{c}$, critical velocity to initiate motion of sediment (based on $0.6 \mathrm{~mm}$-diameter sediment in present study).

\begin{tabular}{cccccccccc}
\hline Case & $S$ & $h(\mathrm{~cm})$ & $U_{m}(\mathrm{~m} / \mathrm{s})$ & $R e$ & $R e_{D}$ & $F r$ & $h / D$ & $U_{m} / u_{c}$ & $u_{*}(\mathrm{~m} / \mathrm{s})$ \\
\hline C9400 & 0.001 & 7.44 & 0.235 & 17500 & 9400 & 0.28 & 1.86 & 0.94 & 0.027 \\
C13500 & 0.002 & 5.85 & 0.338 & 19800 & 13500 & 0.45 & 1.46 & 1.40 & 0.028 \\
\hline
\end{tabular}

\section{Flow characteristics induced by turbulent horseshoe vortex}

\subsection{Time-averaged flow field}

The instantaneous velocity components in the $x$ and $y$ directions are denoted by $u$ and $v$, respectively. The time-averaged flow field was obtained based on a total of 1000 images corresponding to sampling time of $2 \mathrm{~s}$. An additional test examining the effects of sampling time and image number on the time-averaged flow field demonstrates that the present setting can guarantee the convergence of the averaged results (see Appendix A). The time-averaged velocity and its fluctuation in each direction are denoted by $U, V, u^{\prime}$ and $v^{\prime}$, respectively. Figure 2 shows contours of different time-averaged quantities, including the (a) mean streamwise velocity, (b) vorticity, (c) streamwise turbulent intensity and (d) turbulent kinetic energy (TKE). Each quantity is normalized by the water depth (when relevant) and friction velocity $u_{*}$ which is determined from the bed slope and water depth of the incoming flows. The figures in the left and right columns represent cases C9400 and C13500, respectively.

The streamlines superimposed on the flow field show that two turbulent horseshoe vortices (THV) form in front of the cylinder, a primary larger one close to the cylinder (primary THV) and a secondary smaller one further upstream. This two-vortex feature of time-averaged flow field is consistent with previous investigations $[3,18]$. Comparing the left and right figures, it is shown that the streamwise positions of the primary THV center for C9400 and C13500 are located at $x / D=-0.63$ and -0.67 , respectively, which implies for the case with larger $R e_{D}$ the time-averaged primary THV is located further upstream. Apsilidis et al. [12] investigated the time-resolved flow dynamics for a large range of Reynolds number, i.e., $\operatorname{Re}_{D}=(2.9 \sim 12.3) \times$ 
$10^{4}$. They found that the time-averaged topology is $R e_{D}$-invariant regarding its basic features (the presence of two vortices in front of the cylinder), and the streamwise position of the primary vortex varies with $R e_{D}$ and specifically is in the range of $x / D=-0.64 \sim-0.69$. In terms of the number and position of the THV, our present results are consistent with Apsilidis et al. [12], although the range of $R e_{D}$ are different. From inspection of the near-bed flows in Fig. 2, it is seen that the entirety of the measured sections lie within the separated region in front of the cylinder. This is consistent with expectations based on e.g. Fig. 3.4 of Sumer and Fredsøe [4] for the present values of $h / D$. Fig. 2a demonstrates that the magnitude of streamwise velocity is large below the primary THV where the high bed shear stress could induce sediment transport and bed erosion. Fig. $2 b$ indicates the time-averaged vorticity peaks in the center of the primary THV, whereas in the center of the secondary THV the vorticity is relatively small. Dargahi [3] pointed out that the secondary THV is so weak that its contribution to the scour can be negligible. Fig. 2c shows that the largest streamwise turbulent intensity is located beneath the primary THV where the value of the TKE is also large, as shown in Fig. 2d.

From the above description, it can be seen that the primary THV can result in a significant amplification of both mean flow and turbulence statistics and thus may amplify the bed shear stress. As previously pointed out $[16,21]$, the high bed shear stress induced by the THV plays an important role in sediment entrainment and transport in front of the cylinder on a loose bed. Regarding this point, detailed analysis will be given in Section 4. Overall, the present results for the time-averaged flow field are in good agreement with those of previous studies $[12,15,18]$. 
(a)

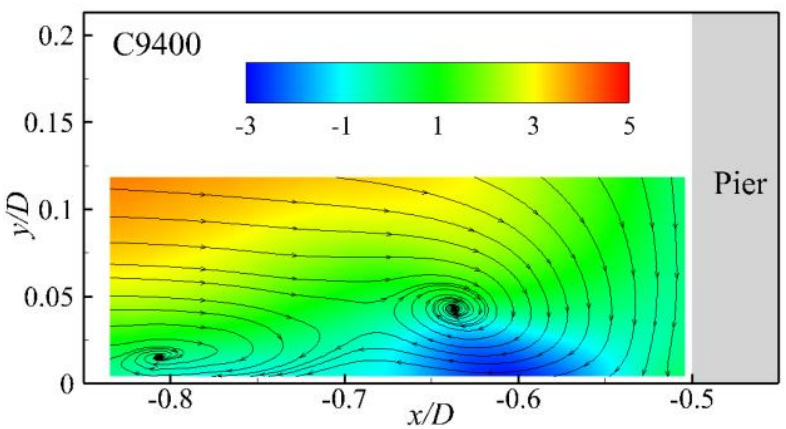

(b)

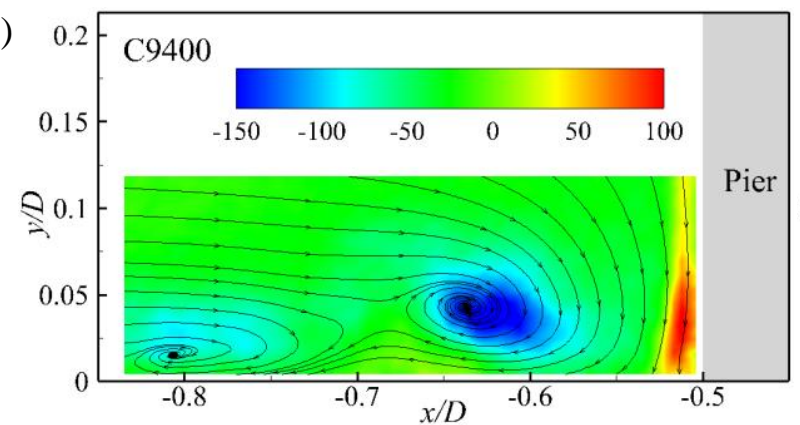

(c)

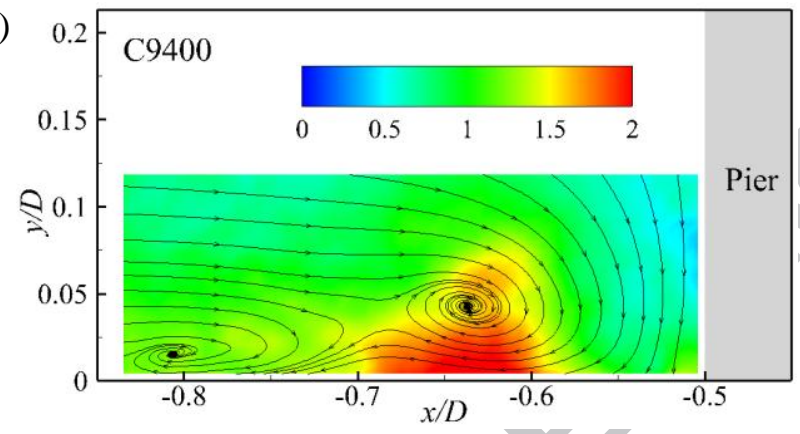

(d)

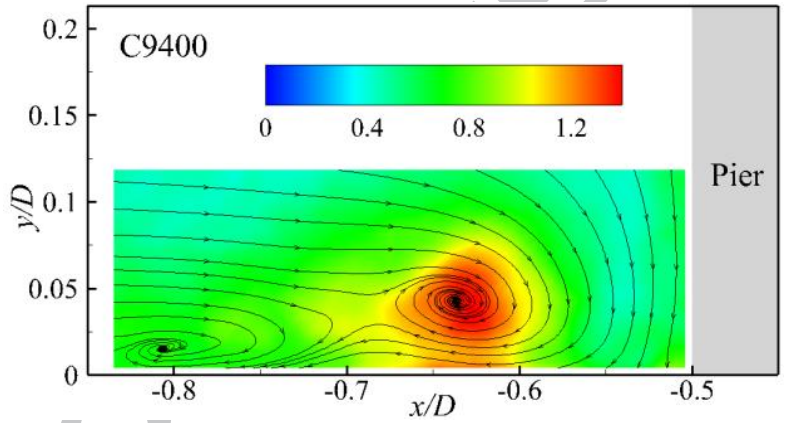

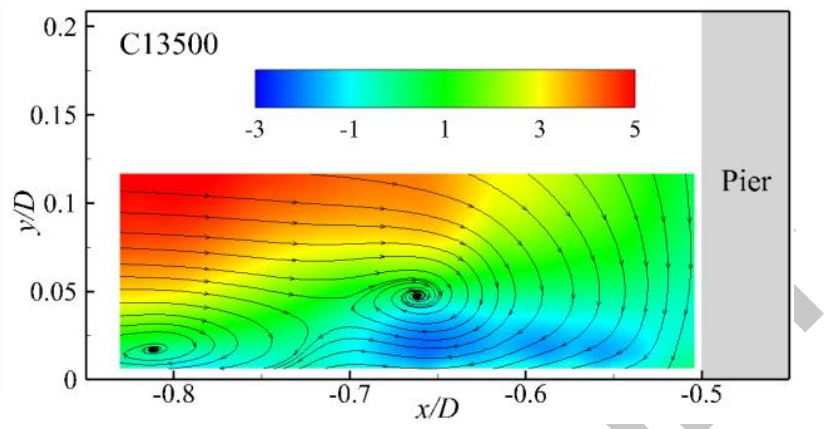
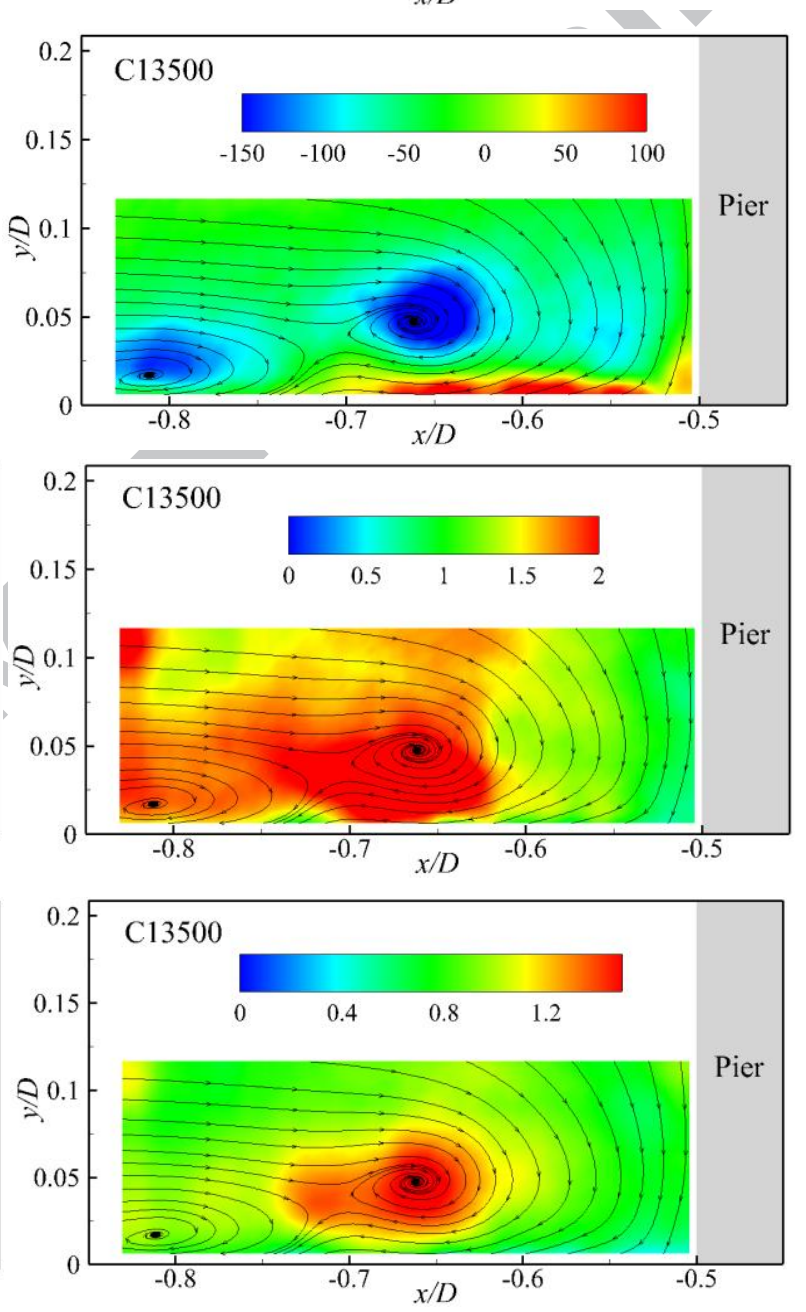

Figure 2 Contours of time-averaged quantities and streamlines at the symmetry plane: (a) streamwise velocity $U^{+}=U / u_{*} ;$ (b) vorticity $\omega^{+}=(\partial U / \partial y-\partial V / \partial x) h / u_{*} ;$ (c) streamwise turbulence intensity $u_{r m s}{ }^{+}=\sqrt{\overline{u^{\prime 2}}} / u_{*}$ and (d) turbulence kinetic energy $k^{+}=0.65\left(\overline{u^{12}}+\overline{v^{12}}\right) / u_{*}^{2}$, where the constant 0.65 is based on Justesen [46].

The overbar $\overline{(\cdot)}$ denotes the time-averaging based on Reynolds decomposition. 


\subsection{Instantaneous flow pattern}

In this Section, in order to explore the dynamics of the THV, the instantaneous flow patterns are comprehensively analyzed. Figure 3 depicts a series of snapshots with instantaneous velocity vectors, demonstrating the process of THV evolution from generation to death. In Fig. 3a, the primary THV is generated in front of the cylinder, and its position is approximately at $x=-0.6 D$. Meanwhile, the presence of the primary THV causes regions of high velocity below. The primary THV then moves upstream and the secondary THV emerges, as shown in Fig. 3b. The primary THV subsequently moves toward the secondary THV and the two then meet and merge (Fig. 3c), producing a single large vortex (Fig. 3d) that moves toward the cylinder (Fig. 3e). Finally, this vortex moves back to $x=-0.6 D$ with decreased strength and size (Fig. 3f), and finally gradually dissipates. The entire process is completed in a short time and then next process is repeated. Several such sequences have been analyzed, which have generally demonstrated similar evolution.

The above described dynamics of the THV in front of the cylinder consequently gives rise to the well-known bimodal oscillations between the so-called zero-flow and back-flow modes, which has been reported by some researchers $[16,18,47,48]$. In this study, we confirm this important feature. Instantaneous velocity vectors colored by the streamwise velocity for case C9400 are depicted in Fig. 4, illustrating these two modes. Fig. 4a exhibits the zero-flow mode, where the primary THV is situated close to the cylinder (i.e., $x=-0.6 D)$ and the shape of its core is close to circular. Alternatively during the back-flow mode shown in Fig. $4 \mathrm{~b}$, the primary THV is situated far from the cylinder, the size of its core is larger, and the shape of its core is close to an ellipsoid. The separation point of the primary THV for the zero-flow mode is around $x=$ $-0.62 D$, whereas this moves to $x=-0.67 D$ during the back-flow mode. For both modes, strong ejections and jet-like flow produced beneath the primary THV induce large amplifications of the bed shear stresses, which is the major cause of sediment transport and erosion in front of a cylinder [48]. 

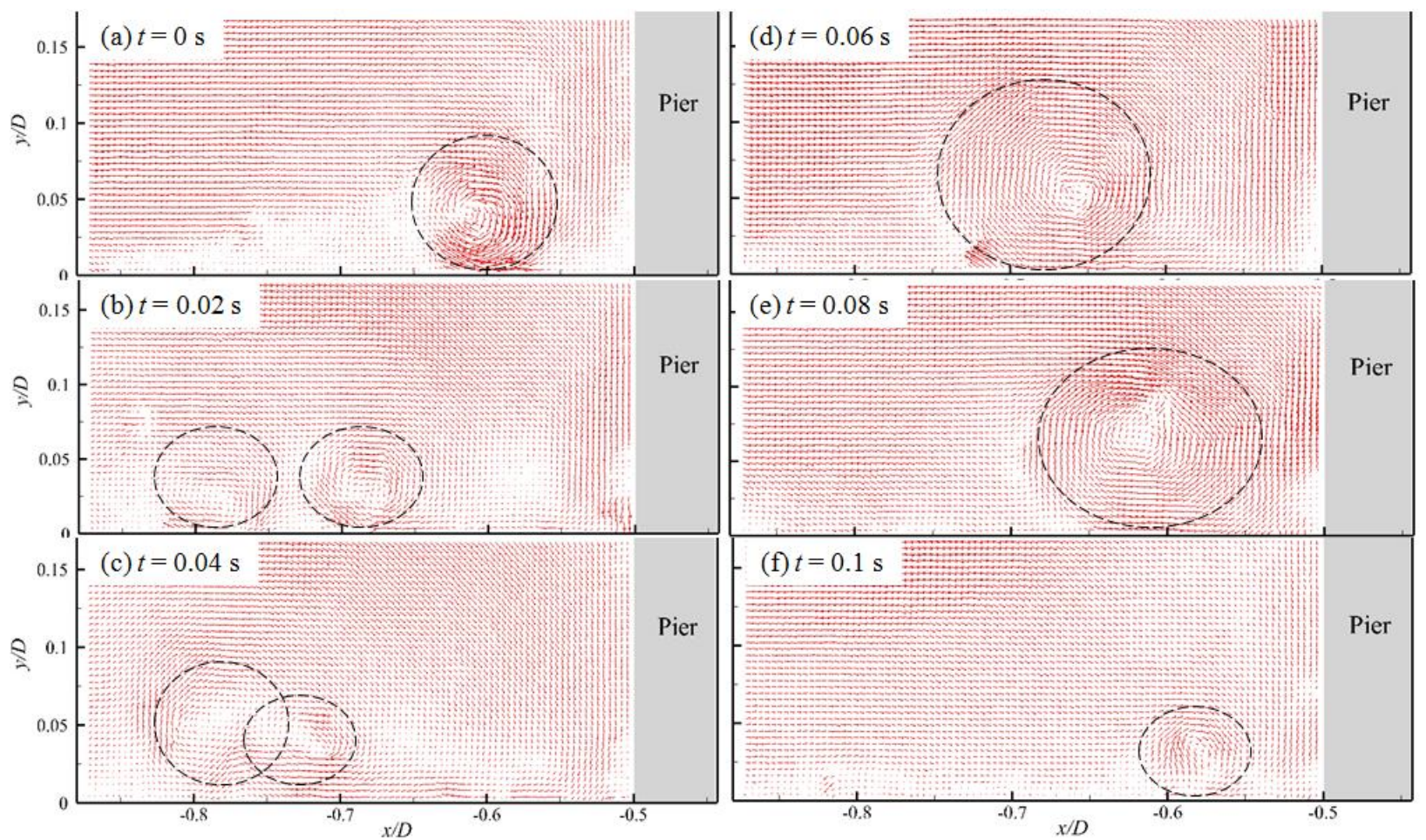

Figure 3 Instantaneous velocity vectors and evolution of turbulent horseshoe vortex from time (a) to (f) for case 9400.

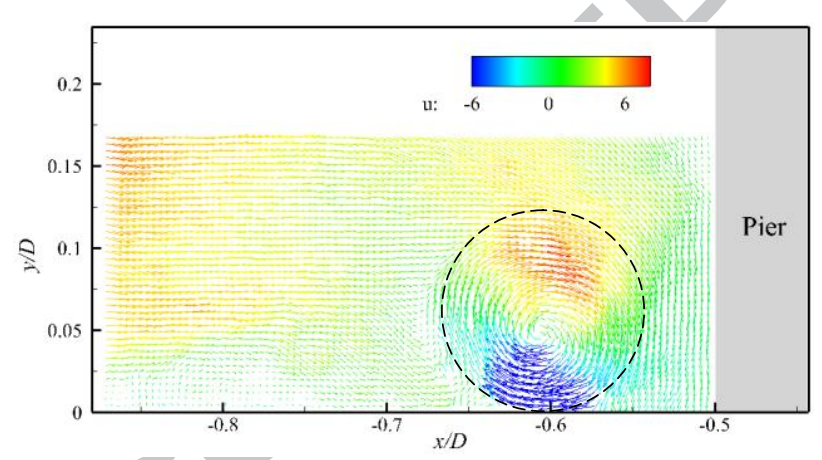

(a)

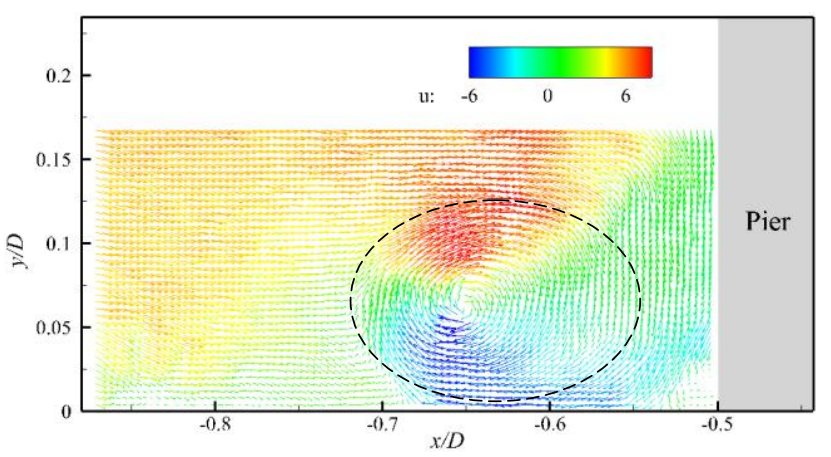

(b)

Figure 4 Instantaneous velocity vectors colored with streamwise velocity for case C9400: (a) zero-flow mode; (b) back-flow mode.

The bimodal dynamics of the THV can be rigorously established by plotting the probability density function (PDF) of the instantaneous streamwise velocity. We extract the instantaneous velocities along a vertical line cutting through the middle of the core of the time-averaged THV, e.g., at $x=-0.63 D$ for C9400 and $x=-0.67 D$ for $C 13500$. The series of histograms shown in Fig. 5 demonstrate that velocities near the wall beneath the THV are clearly bimodal with double-peaked shape for $y / D \leq 0.05$. Particularly for case 
C9400, this double-peaked phenomenon is more apparent than in case C13500. The two peaks of the PDF correspond to $u / u_{m}=0$ and 2 ( $u_{m}$ is the mean velocity at this point), indicating that these two instantaneous velocities frequently occur. This feature is closely related to the flow physics. As mentioned above, this is due to the bimodal oscillations between the zero-flow and back-flow modes, as shown in Fig. 4. The flow beneath the horseshoe vortex exhibits vertical and horizontal jet flow between the two modes, which induces the frequent occurrence of the two instantaneous velocities $\left(u / u_{m}=0\right.$ and 2). The instantaneous velocities for the point away from the bottom, however, exhibit only a single-peaked histogram, and the peak corresponds to $u / u_{m}=1$ implying the flow pattern with mean velocity $u_{m}$ is the most frequent event.

(a)
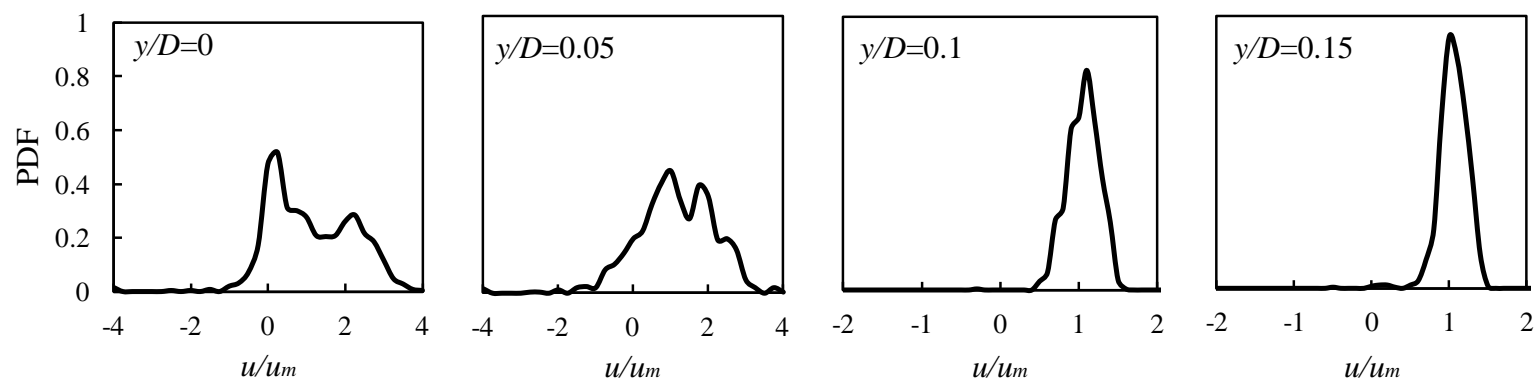

(b)
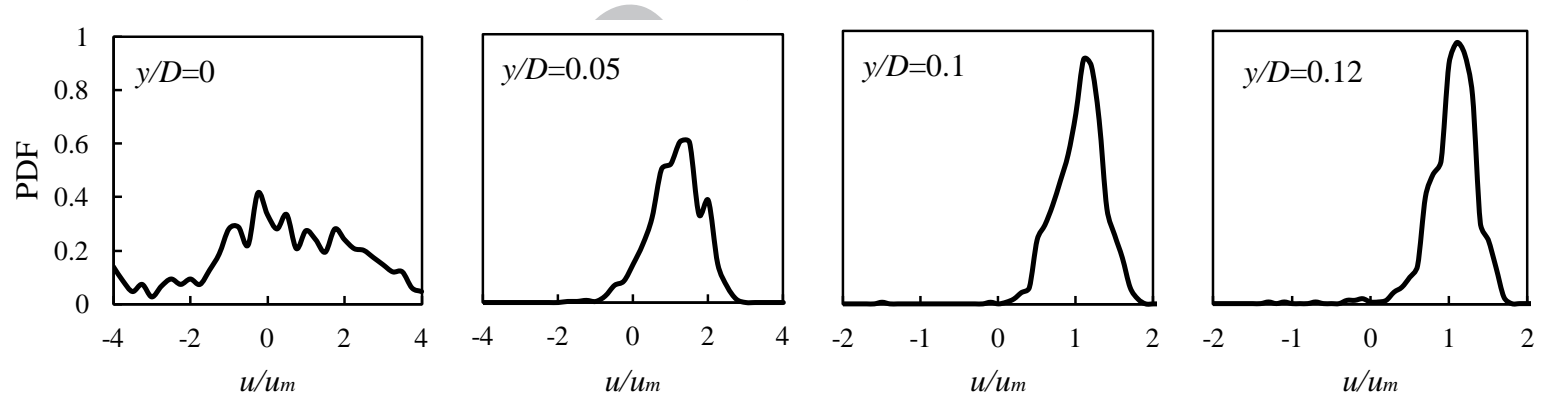

Figure 5 Probability density functions of the instantaneous streamwise velocity $u / u_{m}$ ( $u_{m}$ is the mean velocity at this point) at different points along the line of (a) $x=-0.63 D$ for C9400 and (b) $x=-0.67 D$ for C13500.

\section{Bed shear stress within turbulent horseshoe vortex region}

\subsection{Instantaneous bed shear stress}

As explained above, the dynamics of the THV in front of the cylinder result in high velocities close to the bed and thus significant amplification of bed shear stresses, which is the main reason for the resultant sediment transport and scour processes. In this Section, the bed shear stress within the THV region is analyzed in terms its instantaneous variation and statistical quantities, as well as its probability density function, to identify the contributions of fluctuations to the overall bed shear stress. The bed shear stress $\tau$ on 
the upstream line of symmetry is calculated as follows:

$$
\tau=\rho\left(v+v_{T}\right) \frac{u_{1}}{y_{1}}
$$

where $\rho$ is the water density, $v_{T}$ is the eddy viscosity in the approach flow, and $u_{1}$ and $y_{1}$ are the streamwise velocity and vertical position of the first measurement point above the bed, respectively. A rather similar method was also used by Graf et al. [49], who proposed $\left(v+v_{T}\right)=1.3 \times 10^{-5} \mathrm{~m}^{2} / \mathrm{s}$ under their flow condition. In this paper, maintaining similarity in terms of the non-dimensional quantity $\left(v+v_{T}\right) /\left(U_{m} D\right)$ results in $\left(v+v_{T}\right)=1.8 \times 10^{-6} \mathrm{~m}^{2} / \mathrm{s}$ and $2.6 \times 10^{-6} \mathrm{~m}^{2} / \mathrm{s}$ for cases $\mathrm{C} 9400$ and $\mathrm{C} 13500$, respectively.

According to the above results, the range of the THV dynamic effects in front of the cylinder is approximately $-0.9<x / D<-0.5$, within which the amplifications of instantaneous bed shear stresses are obtained and presented in Fig. 6. The horizontal and vertical axis represent spatial and temporal scales (i.e., $x / D$ and $t$ ), respectively. Here, the stress amplification $\tau_{n}$ is defined as $\tau / \tau_{0}$, in which $\tau_{0}$ is the mean bed shear stress for an undisturbed approaching flow. The black dotted lines in Figs. 6 (a) and (b) represent the streamwise position of mean stress peaks, which are at $x=-0.61 D$ and $-0.66 D$ for case C9400 and C13500, respectively. The regions with large negative values of $\tau_{n}$ that are colored by blue vary temporally around the dashed lines. We further extract the time histories of $\tau_{n}$ at the positions $x=-0.61 D$ for case C9400 and $x=$ $-0.66 D$ for case $\mathrm{C} 13500$, plotted in Fig. 7 , in which the horizontal dashed lines denote the time averaged values of $\tau_{n}$. It can be seen that the fluctuations of the bed shear stress amplification vary dramatically over time, which are similar to the features of turbulence in open-channel flows, e.g., the burr-like peaks due to the turbulent bursting of some small-scale vortices and coherent structures form the boundary layer. Apart from that, it is interesting that the time histories of $\tau_{n}$ fluctuate quasi-periodically around the horizontal dashed lines over relatively large coherent periods of time. This feature is directly linked with the unsteady dynamics and evolution process of the THV as mentioned above. In fact, after filtering the burr-like peaks induced by turbulent bursting, we can clearly see the low-frequency variation of $\tau_{n}$ which is solely due to the action of the THV. This feature is also consistent with LES or DES modeling results $[17,18]$ in which only the large eddies are taken into account. 
(a)

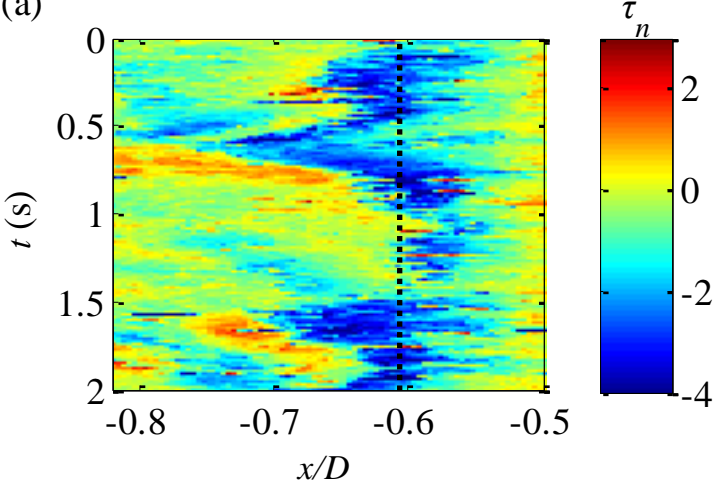

(b)

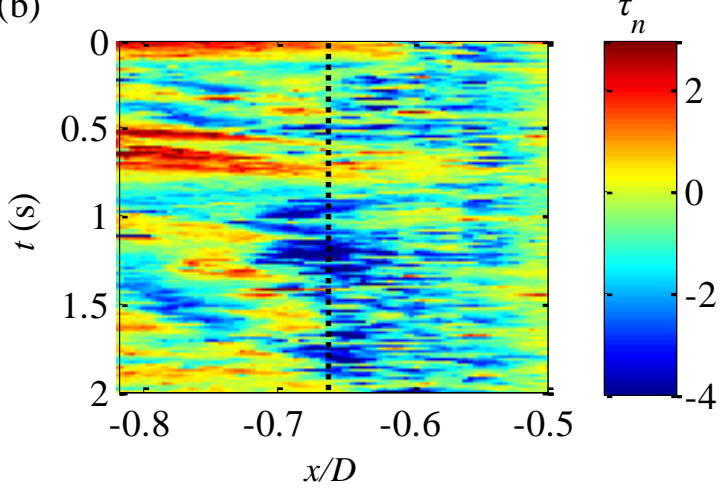

Figure 6 Contours of instantaneous bed shear stress for cases (a) C9400 and (b) C13500.
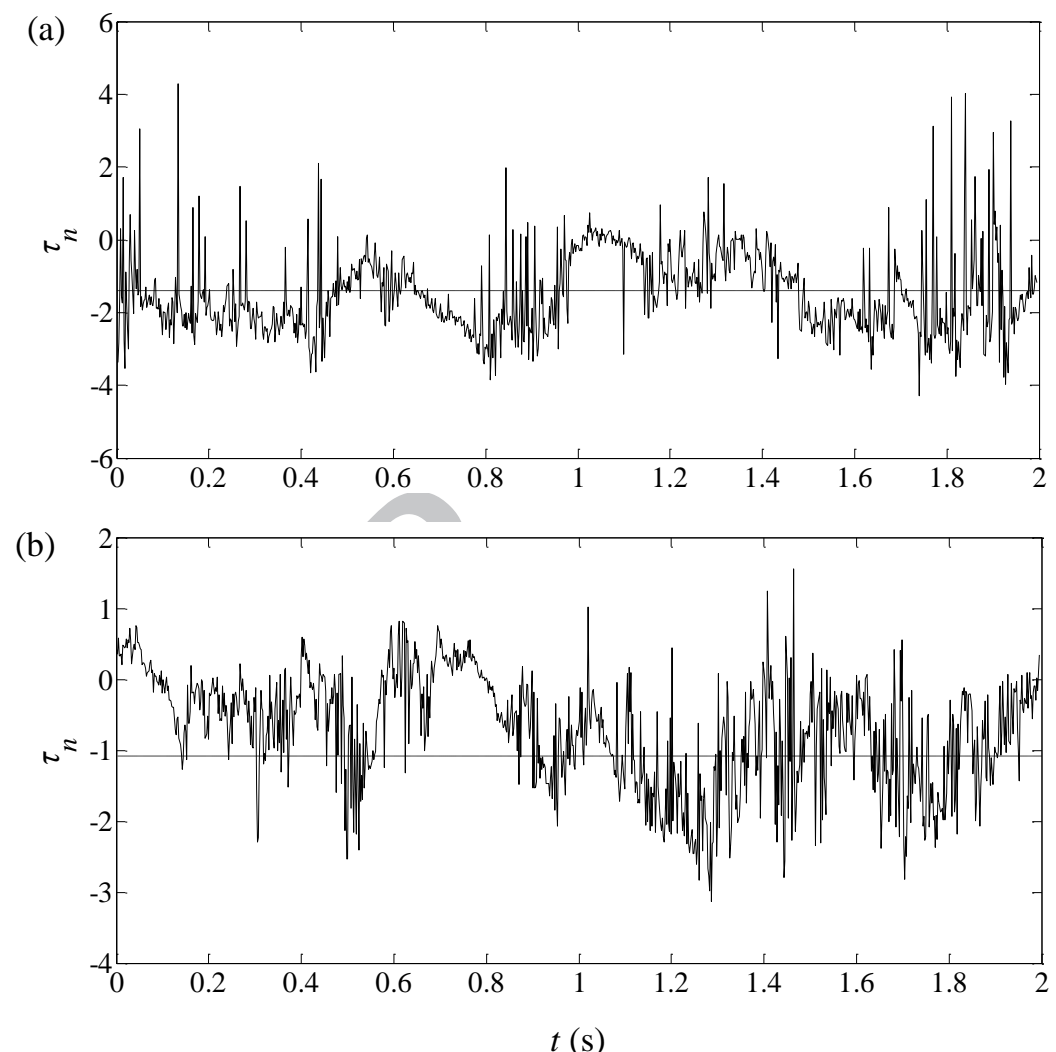

Figure 7 Time histories of instantaneous bed shear stress at the position of (a) $x / D=-0.61$ for C9400 and (b) $x / D=-0.66$ for $\mathrm{C} 13500$.

\subsection{Statistical quantities}

The statistical quantities profiles, including the mean and RMS of the bed shear stress, $\tau_{m}$ and $\tau_{r m s}$, for both C9400 and C13500 cases are shown in Fig. 8. In order to demonstrate the convergence of the statistical result, complementary tests were conducted, and additional data were obtained and utilized for a broader analysis (see Appendix B). Note again that the amplifications in Fig. 8 are normalized with respect to the undisturbed bed shear stress $\tau_{0}$. It shows that both $\tau_{m}$ and $\tau_{r m s}$ beneath the time-averaged primary THV are significantly 
amplified, and both the maximum amplifications tend to decrease as $R e_{D}$ increases. These results agree well with the simulation results obtained by Roulund et al. [21]. In addition, the positions of maximum values irrespective of $\tau_{m}$ or $\tau_{r m s}$ tend to be more upstream with increasing $R e_{D}$, e.g. $x=-0.61 D$ for $\mathrm{C} 9400$ and $x=$ $-0.66 \mathrm{D}$ for $\mathrm{C} 13500$, corresponding to the streamwise position of the primary THV, as discussed above. Further comparison of peak positions between $\tau_{m}$ and $\tau_{r m s}$ demonstrates that instead of exactly overlapping, the peaks in $\tau_{r m s}$ reside upstream. The location of the maximum $\tau_{r m s}$ is influenced by the secondary THV located immediately upstream of the primary THV. These two vortices strongly interact, thereby inducing large turbulence in the bed shear stress between their centers.

The dashed horizontal lines in Fig. 8 represent the critical mean bed shear stress for case C9400 (clear-water condition) along the $z=0$ plane, beyond which sediment erosion occurs. The critical shear stress $\tau_{c}$ is again based on non-cohesive sediment with diameter of $0.6 \mathrm{~mm}$ in the present study. For comparative purposes, we use the absolute value (i.e., $\left|\tau_{r m s}\right| \tau_{0} \mid$ ) in Fig 8 b. It shows that the scour area based on $\tau_{m}$ (Fig. 8a) is smaller than that based on $\tau_{r m s}$ (Fig. 8b), which implies that the scour area could be underestimated if it is only based on the mean bed shear stresses and neglecting the fluctuations.

In order to quantify the contribution of the bed shear stress fluctuations, we define the magnitude of the relative bed shear stress fluctuation $I=\left|\tau_{r m s} / \tau_{m}\right|$. Figure 9 presents the distribution of $I$ along the $x$ direction in the range of $-0.64<x / D<-0.56$ corresponding to the expected scour region for C9400. It demonstrates that the value of $I$ is between 0.6 and $1.2(=O(1))$, illustrating that the fluctuations of bed shear stress are comparable to those of the mean. Cheng et al. [38] pointed out that when $I>0.3$ the influence of bed shear stress fluctuation to sediment transport is great, and the sediment transport rate based only on the mean bed shear stress, neglecting its fluctuation, is rather under-predicted. This point is discussed in detail in Section 5.

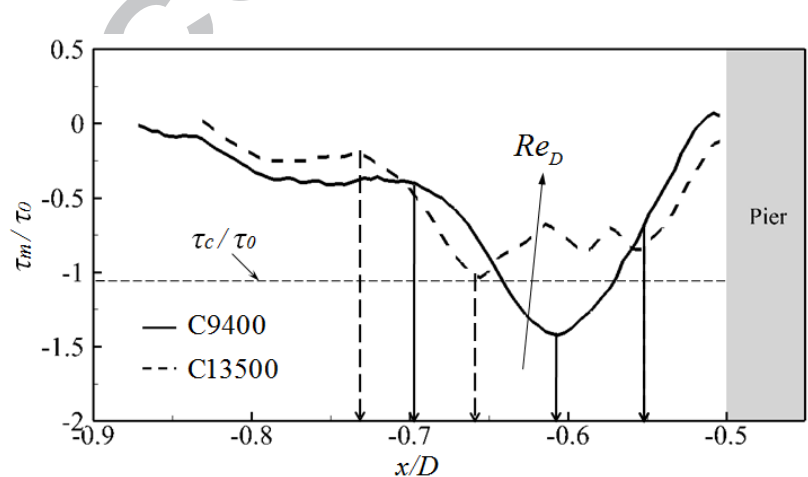

(a)

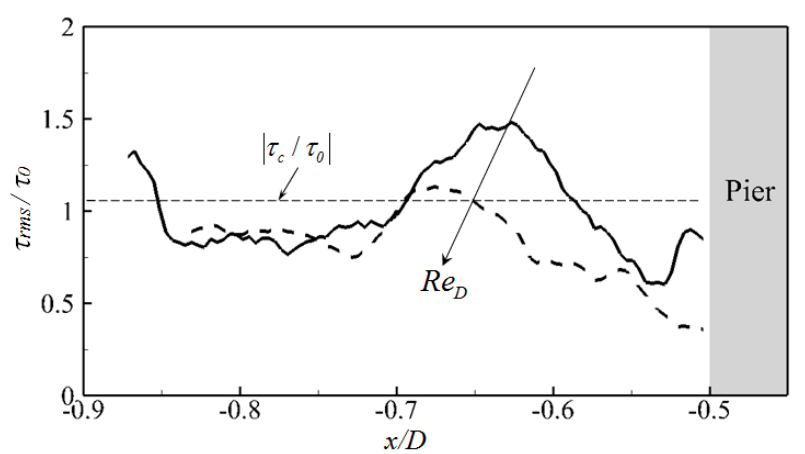

(b)

Figure 8 Distribution of bed shear stress statistics along the symmetry upstream of the cylinder: (a) Mean bed shear stress; (b) RMS of the bed shear stress. 


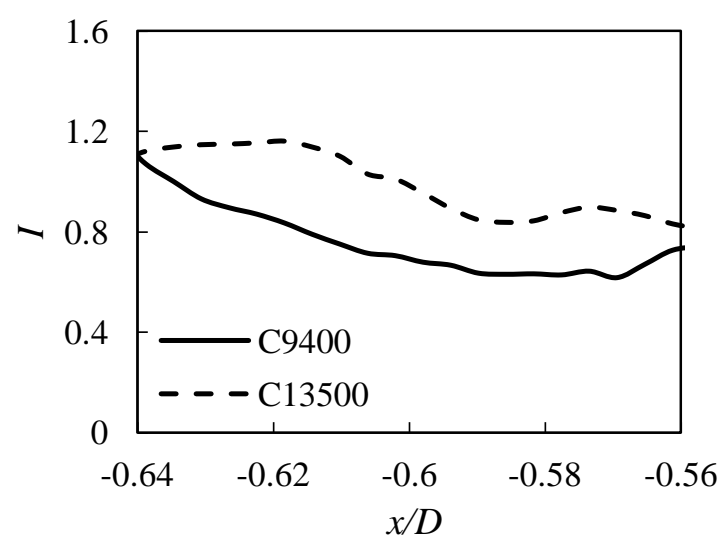

Figure 9 Distribution of relative bed shear stress fluctuation $I$ along $x$ direction.

\subsection{Probability density function}

Regarding the fluctuation of the bed shear stress, which may not be negligible in the calculation of cylinder scour, Chang et al. [32] pointed out that the contribution of the bed shear stress fluctuation is necessarily taken into account by a certain weight due to the strong turbulence. Cheng et al. [38] reported that the probability density function (PDF) of the fluctuating bed shear stress at a point under the influence of strong turbulence can be represented by the log-normal function, as follows

$$
f\left(\tau_{I}\right)= \begin{cases}\frac{1}{\sqrt{2 \pi\left(1+I^{2}\right) \tau_{I}}} \exp \left[-\frac{\left(\ln \tau_{I}+\ln \sqrt{1+I^{2}}\right)^{2}}{2 \ln \left(1+I^{2}\right)}\right] & \text { for } \tau_{I}>0 \\ 0 & \text { for } \tau_{I} \leq 0\end{cases}
$$

where $\tau_{I}=\tau / \tau_{m}=$ normalized bed shear stress. From Eq. (2), it can be seen that for small $I$-values, the PDF of the bed shear stress degenerates to a Gaussian distribution. Eq. (2) has been validated by the experiment of Sumer et al. [40] concerning uniform and unidirectional flows, however, for rapidly varied flows with reversal vortices in front of a cylinder, the applicability of Eq. (2) is, as yet, unknown.

In Fig. 10 the PDFs of bed shear stress based on Eq. (2) are shown all across the profile, and the large values of $f\left(\tau_{I}\right)$, which are colored by red, are clearly within the range $\tau_{I}=0-1$. For the sake of comparison between experimental and calculated results, we extract the PDFs of the bed shear stress at several typical points, $x=-(1.6,0.7,0.61,0.55) D$ for $C 9400$ and $x=-(1.6,0.73,0.66,0.55) D$ for $\mathrm{C} 13500$, which are presented separately in Fig. 11. Additionally, the calculated PDFs from Eq. (2) are also included as the full lines. The four points for each case are sequenced by location as (1) the region of undisturbed flow away 
from THV, (2) the upstream edge of the THV, (3) inside the THV, and (4) downstream of the THV. The final three positions are marked with the arrows shown on Fig. 8a. On Fig. 11, we can see that the calculated results agree well with experiments at $x=-1.6 D$, which is uninfluenced by the THV, and the distribution of the PDF is close to Gaussian. For the region influenced by the vortex, however, the PDFs tend to be log-normal distributed with clear positive skewness. More importantly, the PDFs at the points $x=-0.61 D$ and $-0.66 D$ show double-peaked distributions that are divergent from the log-normal distribution, Eq. (2). The double-peaked shape of the PDF can be explained by the aforementioned bimodal oscillations in the THV region. Therefore, although Eq. (2) performs well for uniform flow as validated by Cheng et al. [38] and confirmed again here, and even for the turbulent flow very close to the vortex, it is not applicable for the flow within the THV region in front of the cylinder.

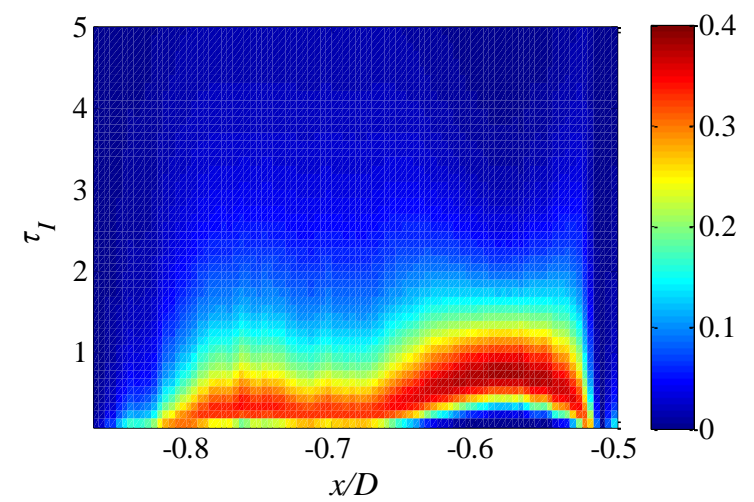

(a)

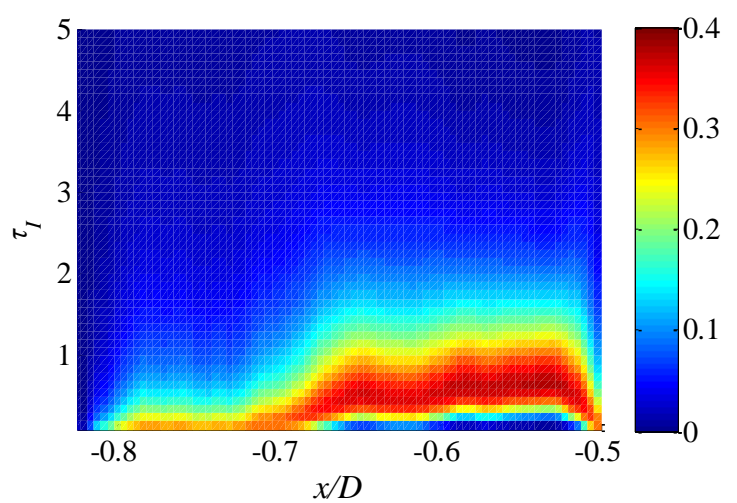

(b)

Figure 10 Contour of PDF of bed shear stress calculated by Eq. (2) for (a) C9400 and (b) C13500. 

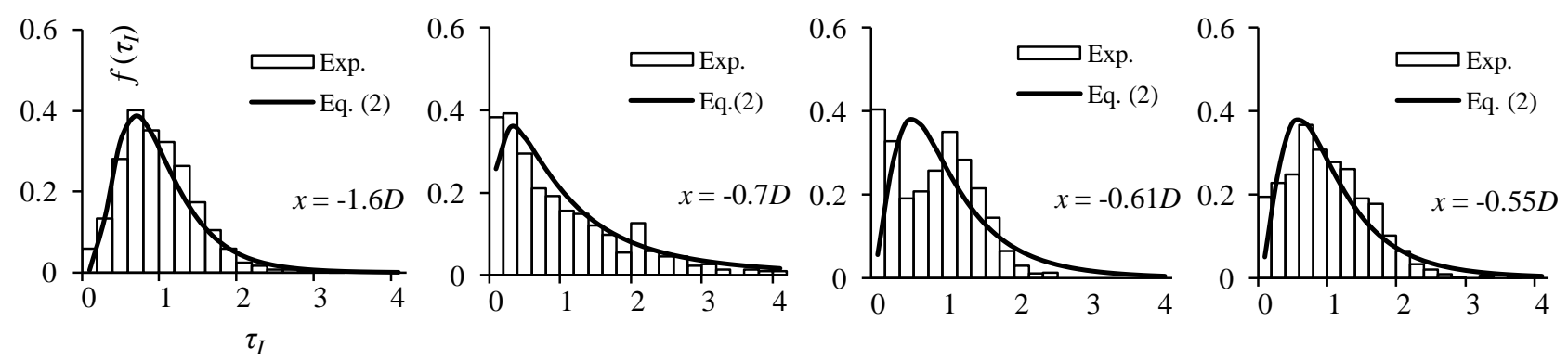

(a)
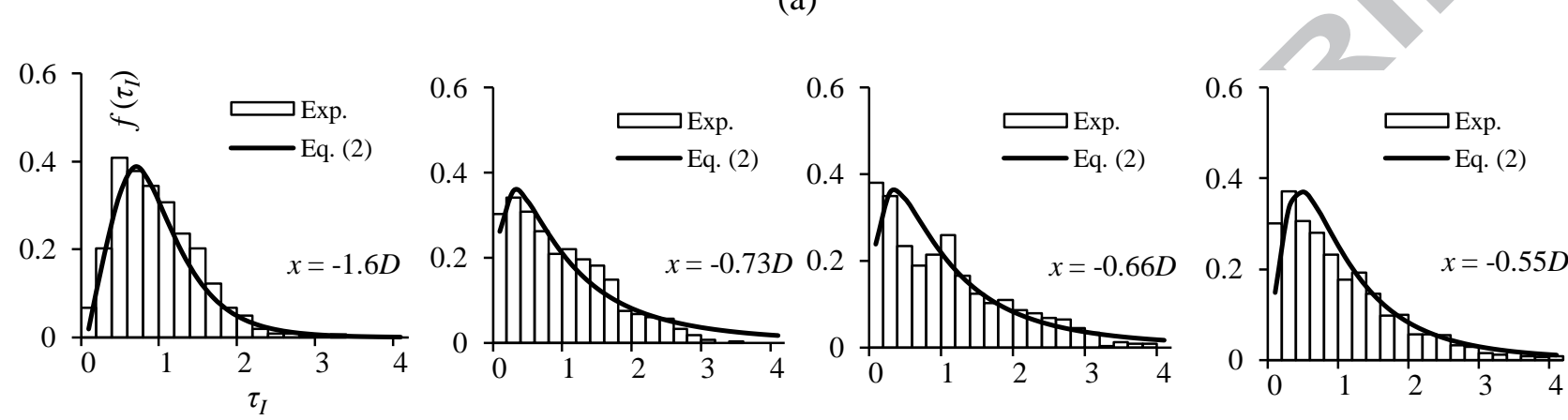

(b)

Figure 11 Comparisons of experimental and calculated PDFs of bed shear stress at representative locations relative to THV: (a) C9400; (b) C13500.

\section{Influence of bed shear stress fluctuation on sediment transport}

\subsection{Comparison of sediment transport rate}

The fluctuations of bed shear stresses inside the THV region, as analyzed above, are almost the same order as the mean bed shear stresses. Thus, the sediment transport rate based on traditional methods, i.e. only considering the mean bed shear stress and neglecting its fluctuation, could be under-predicted. In this Section, the under-prediction is quantified by taking the influence of fluctuations into consideration. Most of the approaches for estimating sediment transport rates typically assume that the (bed load) sediment flux is proportional to $\left(\tau-\tau_{c}\right)^{c / 2}$ (e.g., $c=3$ in van Rijn's equation [50]). It should be noted that the major mode of sediment transport in front of the cylinder is the bed load transport according to previous relevant results [3, 48], particularly in the present cases under consideration. Thus, the dimensionless instantaneous and mean sediment transport rates, $\phi$ and $\phi_{m}$, based on instantaneous and mean bed shear, $\tau$ and $\tau_{m}$, can be respectively calculated by

$$
\phi=\frac{1}{U^{3} T \rho^{3 / 2}} \int_{0}^{T}\left(\tau-\tau_{c}\right)^{3 / 2} d t
$$




$$
\phi_{m}=\frac{1}{U^{3} \rho^{3 / 2}}\left(\tau_{m}-\tau_{c}\right)^{3 / 2}
$$

where $T=2 \mathrm{~s}$ is used, corresponding to the measurement duration. Figure 12 compares the distributions of instantaneous and mean sediment transport rates, in which the solid and dotted lines represent $\phi$ and $\phi_{m}$, respectively. This figure demonstrates that the value of $\phi$ is markedly larger than that of $\phi_{m}$. More specifically, the value of the predicted instantaneous sediment transport rate is approximately double that for the mean sediment transport rates (i.e. $\phi \approx 2 \phi_{m}$ ). On the other hand, the sediment transport rate based on the mean bed shear stress is (apparently) comparatively underestimated. Additionally, for the clear-water conditions of case C9400, Fig. 12a shows that the mean predicted sediment transport rate away from the cylinder is zero indicating that the sediment entrainment would not be expected occur. Alternatively, the instantaneous sediment transport rate at corresponding locations is not zero, which implies that the initiation of sediment motion may also be underestimated by the mean bed shear stress.

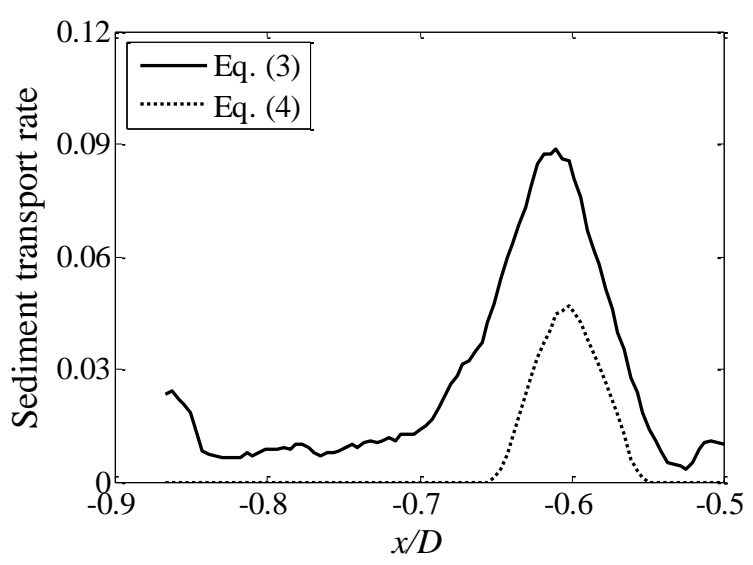

(a)

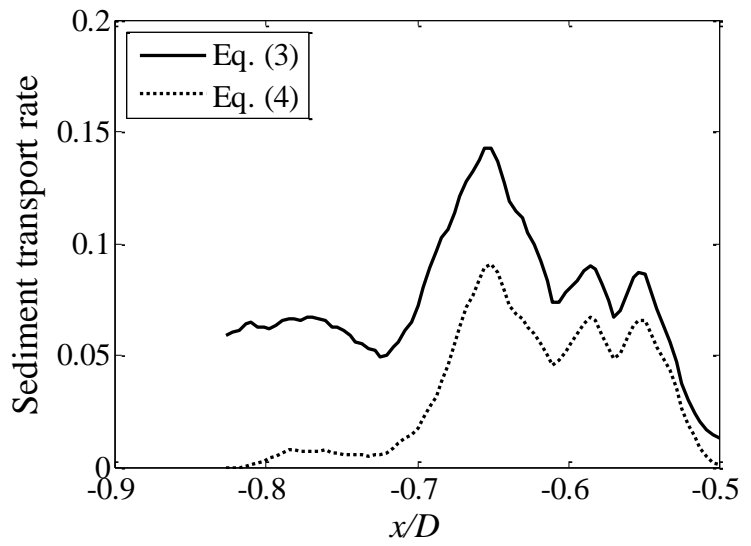

(b)

Figure 12 Comparisons of sediment transport rates based on instantaneous and mean bed shear stresses for (a) C9400 and (b) C13500.

The spatial averaged flux of sediments entrained from the bed in front of the cylinder can be obtained by integrating the local sediment transport rates over the bed area. In the present study, the spatial averaged sediment transport rates are calculated based on three different approaches: (i) both sediment transport rate and initiation of sediment based on the instantaneous bed shear stress, $\langle\phi\rangle$; (ii) the sediment transport rate based on the instantaneous bed shear stress and the initiation of sediment based on mean bed shear stress, 
$\left\langle\phi^{\prime}\right\rangle$; and (iii) both sediment transport rate and initiation of sediment based on the mean bed shear stress, $\left\langle\phi_{m}\right\rangle$. These are respectively described by the following dimensionless quantities:

$$
\begin{aligned}
\langle\phi\rangle & =\frac{1}{D U^{3} \rho^{3 / 2}} \int_{X, \tau>\tau_{c}} \int_{0}^{T}\left(\tau-\tau_{c}\right)^{3 / 2} d t d x \\
\left\langle\phi^{\prime}\right\rangle & =\frac{1}{D U^{3} \rho^{3 / 2}} \int_{\substack{X_{m}, \tau_{m}>\tau_{c} \\
\tau>\tau_{c}}} \int_{0}^{T}\left(\tau-\tau_{c}\right)^{3 / 2} d t d x \\
\left\langle\phi_{m}\right\rangle & =\frac{1}{D U^{3} \rho^{3 / 2}} \int_{X_{m}, \tau_{m}>\tau_{c}} \int_{0}^{T}\left(\tau_{m}-\tau_{c}\right)^{3 / 2} d t d x
\end{aligned}
$$

where $X$ and $X_{m}$ are the bed areas where $\tau>\tau_{c}$ and $\tau_{m}>\tau_{c}$, respectively. The results calculated by Eqs. (5a) (5c) are listed in Table 2. Since the case of C13500 is in the live-bed scour regime, the value of $\langle\phi\rangle$ is almost equal to that of $\left\langle\phi^{\prime}\right\rangle$. By contrast, for case C9400 (clear-water scour conditions), there exists a difference between $\langle\phi\rangle$ and $\left\langle\phi^{\prime}\right\rangle$, as analyzed above from Fig. 12a, resulting in underestimation of sediment initiation. Additionally, the values of $\langle\phi\rangle$ are clearly larger than $\left\langle\phi_{m}\right\rangle$; in particular for clear-water conditions the ratio of $\langle\phi\rangle$ to $\left\langle\phi_{m}\right\rangle$ is up to 3.38. In other words, the sediment transport rate based on the mean bed shear stress could be remarkably underestimated by as much as $70 \%$. Therefore, combining the above comparative analysis and the values listed in Table 2, it is again confirmed that the sediment transport rate in front of the cylinder may be significantly underestimated when neglecting the influence of turbulent bed shear stress fluctuations.

Table 2 Comparisons of sediment transport rates based on different methods.

\begin{tabular}{cccccc}
\hline Case & $\langle\phi\rangle\left(\times 10^{-6}\right)$ & $\left\langle\phi^{\prime}\right\rangle\left(\times 10^{-6}\right)$ & $\left\langle\phi_{m}\right\rangle\left(\times 10^{-6}\right)$ & $\langle\phi\rangle /\left\langle\phi_{m}\right\rangle$ & $\left\langle\phi^{\prime}\right\rangle /\left\langle\phi_{m}\right\rangle$ \\
\hline C9400 & 12.08 & 7.72 & 3.09 & 3.88 & 2.50 \\
C13500 & 30.43 & 29.53 & 13.60 & 2.24 & 2.17 \\
\hline
\end{tabular}

\subsection{New sediment transport model incorporating the effect of bed shear stress fluctuations}

The ratio of instantaneous sediment transport rate normalized by the mean sediment transport rate, 
$\phi_{n}=\phi / \phi_{m}$, is related to the relative bed shear stress fluctuation $I$ [39]. Sumer et al. [40] provided an empirical relationship between $\phi_{n}$ and $I$ for uniform open-channel flows without a cylinder. In the present study, the data of $\phi_{n}$ with $I$ based on our cylinder-flow experiments are plotted in Fig. 13a, in which the data of Sumer et al. [40] are also included to consistently extend the applicable conditions. It can be seen that $\phi_{n}$ generally increases with increasing $I$, but that the rate of increase is different for different situations, which means that the influence of $I$ on $\phi_{n}$ varies with condition.

We obtain the quantitative relationship between $\phi_{n}$ and $I$ by fitting the experimental data from the present study as well as the previous study of Sumer et al. [40], as follows:

$$
\phi_{n}=\exp [m(I-0.44)] \quad R^{2}=0.876-0.947
$$

where $R^{2}$ is the correlation coefficient, and $m$ is a tuning coefficient quantifying the influencing degree of $I$ to $\phi_{n}$. A total number of 90 data points from both present experiments and Sumer et al. [40] were used to obtain Eq. (6a). Fig. 13a shows that the influence for the case from Sumer et al. [40] $(m=6.969)$ is of the largest, followed by cases C9400 $(m=2.481)$ and C13500 ( $m=0.733)$. In other words, the effect of $I$ on $\phi_{n}$ for case C13500 is lower than that for case C9400. The possible reason is that case C13500 is in a live-bed scour condition (i.e., sediments supply from upstream). As mentioned above, in a live-bed condition, sediment motion is not only driven by the THV but also by the turbulence bursting from the boundary layer [19]. The contribution of THV-induced turbulence to sediment transport is probably lower in live-bed conditions, and thus the effect of $I$ is correspondingly lower.

Assuming the cylinder Reynolds number in the case from Sumer et al. [40] as zero (i.e., $R e_{D}=0$ ), it is found that $m$ tends to decrease with increasing $R e_{D}$. We further obtain the relationship of $m$ on $R e_{D}$ by fitting the data plotted in Fig. 13b, as follows:

$$
m=6.969 \exp \left(-1.172 \times 10^{-4} R e_{D}\right) \quad R^{2}=0.928
$$

Here, Eq. (6b) is an exponential function. When $R e_{D}=0, m=6.969$, and hence Eq. (6a) simplifies to that recommended by Sumer et al. (2003). When $\operatorname{Re}_{D} \rightarrow \infty$, then $m \rightarrow 0$, and accordingly $\phi_{n} \rightarrow 1$ based on Eq. (6a). It should be noted that the range of application for Eq. (6) is $I=0.44-1.2$.

A plot summarizing the experimental versus calculated (utilizing Eq. (6)) sediment transport rate is 
depicted in Fig. 14. As can be seen, the sediment transport model proposed above does a consistently good job of predicting the sediment transport rate with almost all data generally near the line of perfect agreement (full line) in Fig. 14. It is emphasized that Eq. (6) can be used to modify the calculation of sediment transport rate not only for uniform open-channel flow but also for the vortex flow in front of the cylinder.

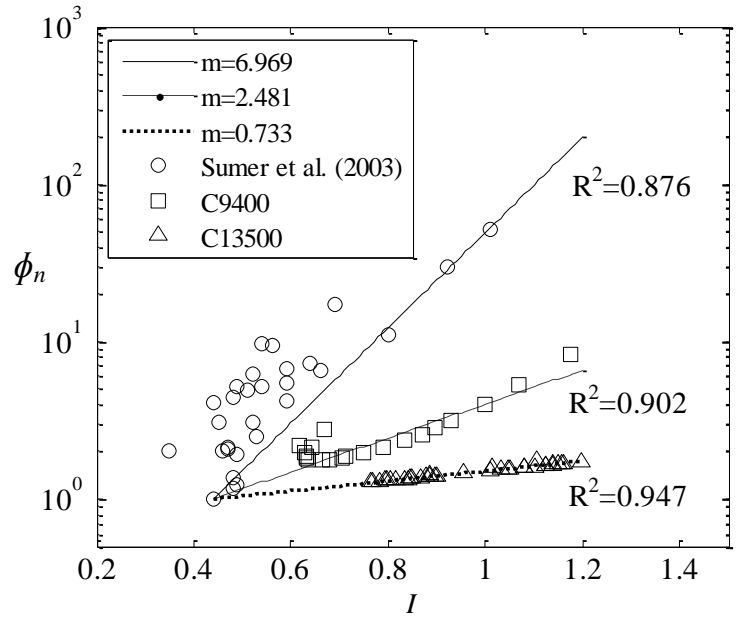

(a)

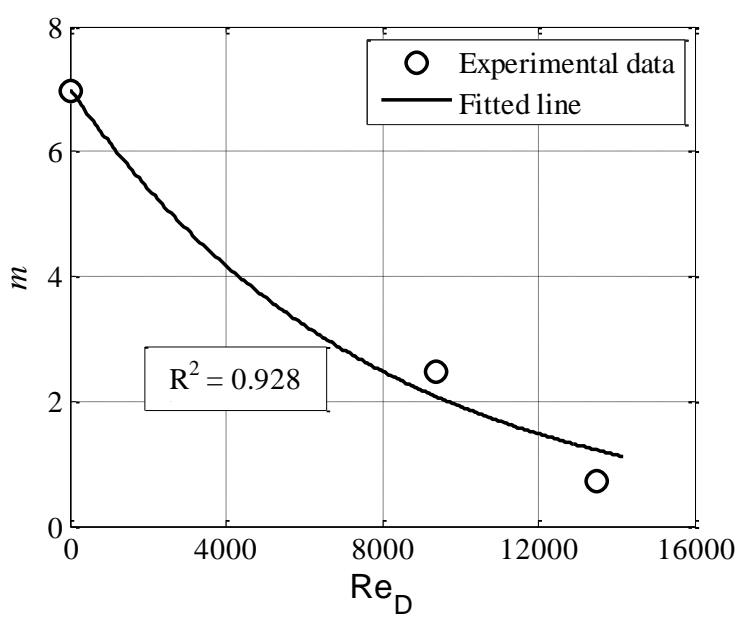

(b)

Figure 13 Relationship between relative sediment transport rate and relative bed shear stress fluctuation: (a) $\phi_{n}$ versus $I$; (b) $m$ versus $R e_{D}$.

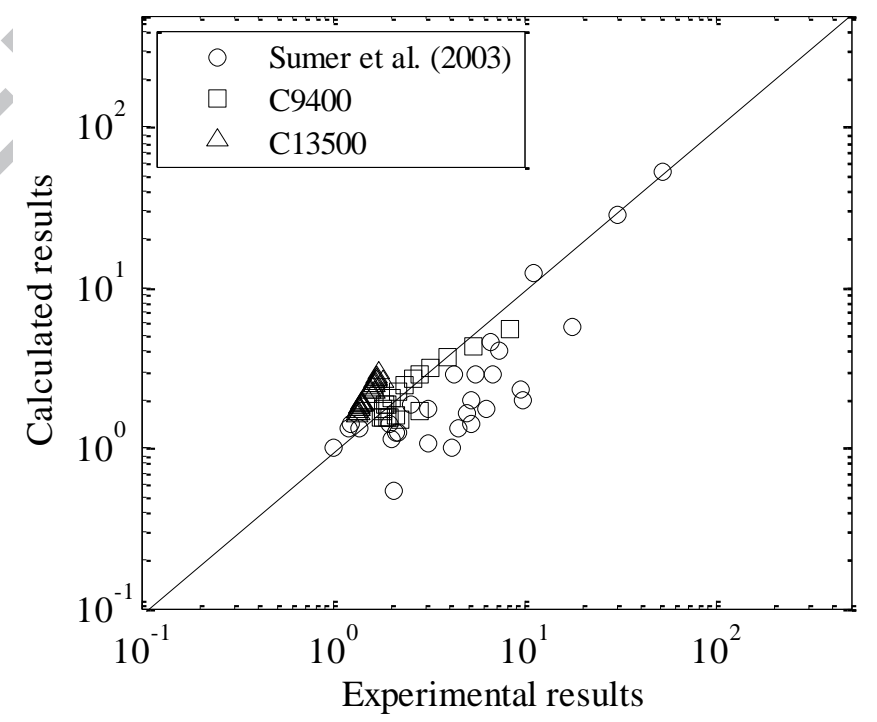

Figure 14 Comparison of calculated (by Eq. (6)) and experimental sediment transport rates. 


\subsection{Validation of proposed sediment transport model}

In this Section, the scour rates at the initial scour stage in front of a circular cylinder were predicted and compared with available results from Escauriaza and Sotiropoulos [48] who used DES to simulate the flow past a circular cylinder mounted on a mobile bed, which corresponds to the case studied experimentally by Dargahi $[3,36]$. This validation case is selected because (i) the initial stage of scour starting from a flat bed for the first $5 \mathrm{~s}$ is provided [48] and (ii) the corresponding mean and RMS values of bed shear stress $\left(\tau_{m}\right.$ and $\left.\tau_{r m}\right)$ are also given (see Fig. 13 of Dargahi [3]), which can be used to calculate sediment transport and scour. In this case, the cylinder diameter is $D=0.15 \mathrm{~m}$ and the approach mean flow velocity is $0.26 \mathrm{~m} / \mathrm{s}$, which corresponds to the cylinder Reynolds number $R e_{D}=39,000$. The sediment is uniform with mean diameter of $0.36 \mathrm{~mm}$. The ratio of the mean bed shear stress to the critical shear stress for sediment initiation is $\tau_{m} / \tau_{c}=$ 0.85 corresponding to a clear-water scour condition.

The change of bed elevation in front of the cylinder is calculated based on the sediment continuity (Exner) equation:

$$
r=\frac{\partial y_{b}}{\partial t}=-\frac{1}{1-\lambda} \frac{\partial q_{s}}{\partial x}
$$

where $r$ is the scour rate, $y_{b}$ is the bed elevation, $\lambda=0.4$ is the bed porosity, and $q_{s}$ is the sediment transport rate (only bed load). Eq. (7) can be discretized into the following finite-difference form:

$$
r=\frac{\partial y_{b}}{\partial t}=-\frac{1}{1-\lambda} \frac{q_{s}^{i+1}-q_{s}^{i}}{\Delta x}
$$

where $\Delta x$ is the spatial step. To validate the new proposed sediment transport model, $q_{s}$ on the right side of Eq. (8) is calculated by Eq. (6) in which the relation between $\phi$ and $q_{s}$ is

$$
\phi=\frac{q_{s}}{\sqrt{g(s-1) d_{50}^{3}}}
$$

The calculation procedure is: 1) the sediment transport rate $\phi_{m}$ is calculated by Eq. (4) in which the data of $\tau_{m}$ is from Dargahi [36]; 2) the modified sediment transport rate $\phi$ is then calculated by Eq. (6) in which $I=\left|\tau_{r m s} / \tau_{m}\right|$;3) $\phi$ is inserted into Eq. (9) to get $q_{s}$; 4) the scour rate $r$ is finally calculated from Eq. (8) utilizing MATLAB.

We assume that the scour depth increases linearly at the initial stage of $t=0-5 \mathrm{~s}$ [51], hence the scour rate is constant during the initial $5 \mathrm{~s}$. Correspondingly, the scour rate from the DES modeling result [48] can be approximately defined as $r=d_{s} / T_{D}$ where $T_{D}=5 \mathrm{~s}$ and $d_{s}$ is the scour depth at $5 \mathrm{~s}$. The scour rates along 
the symmetry plane upstream of the cylinder at the initial stage are presented in Fig. 15, in which the scour rates are normalized by the ratio of cylinder diameter to time duration (i.e., $D / T_{D}$ ), and the positive and negative values represent bed deposition and scour, respectively. The red line is the scour rate predicted by the proposed new sediment transport model, i.e., Eq. (6), denoted as $r_{s}$. For comparison, the result predicted by the conventional sediment transport method, i.e., Eq. (4), was also depicted in Fig. 15 by the blue line, denoted as $r_{s m}$. It clearly shows that the profile of $r_{s}$ generally agrees well with the DES result especially for the maximum value of deposition and its position, while the values of $r_{s m}$ are generally smaller than the DES result. It therefore indicates that the initial scour rates may be under-predicted by the conventional sediment transport equation only accounting for mean bed shear stress, and the new proposed sediment transport model incorporating the influence of bed shear stress fluctuation improves the prediction of bridge scour at the initial stage.

Additionally, from the tendency of the profiles, the present results are qualitatively consistent with the DES results since both show the predominant phenomenon of scour and deposition in front of the cylinder. As discussed above, the scour in front of the cylinder is mainly due to the existence of the primary THV which induces significant amplification of bed shear stress below, and thus the position of the scoured region coincides with the streamwise position of the time-averaged primary THV. The sediment transported by the return flow of the primary THV is accumulated upstream causing deposition upstream of the scour area.

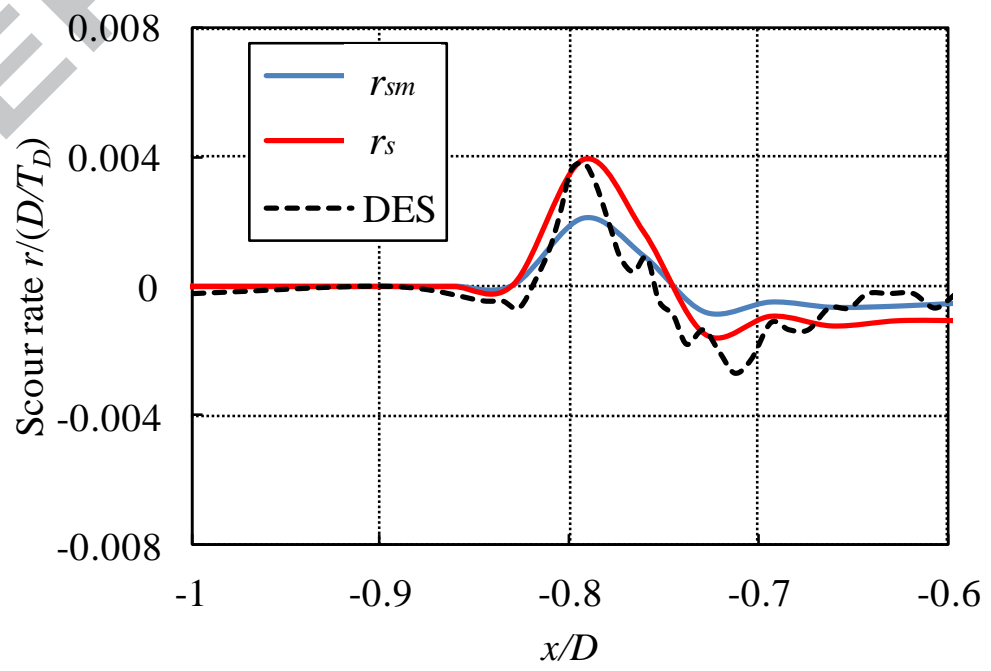

Figure 15 Comparison of initial scour rate in front of a cylinder based on different sediment transport equations (Eqs. (4) and (6)). 


\section{Conclusions}

The conclusions drawn from the present study are summarized as follows:

(1) Two time-averaged turbulent horseshoe vortices (THVs) are identified in front of the cylinder with the primary THV tending to be further upstream for the larger $R e_{D}$ case. The primary THV results in a significant amplification of both mean flow and turbulence statistics (i.e., turbulent intensity and turbulent kinetic energy) below. The dynamics of the instantaneous THV exhibit a quasi-periodic process from generation to death within the region of $-0.9<x / D<-0.5$.

(2) The variation of the instantaneous bed shear stress is directly associated with the dynamics of the THV. Both $\tau_{m}$ and $\tau_{r m s}$ underneath the primary THV are significantly amplified, and both the maximum amplifications tend to decrease as $R e_{D}$ increases. The fluctuations of the bed shear stress are comparable to those of the mean. The probability density function of the bed shear stress is characterized by a double-peaked distribution within the THV region and cannot be represented by the normally used log-normal distribution for uniform open channel flows.

(3) Based on comparisons where turbulent fluctuations are, or not included, it appears that the sediment transport rates based on mean bed shear stress may be underestimated. The comparisons of spatial averaged sediment transport rates show that the flux of sediment based on mean bed shear stresses could be remarkably underestimated by as much as $70 \%$ for clear-water scour conditions.

(4) A new sediment transport model incorporating the influence of bed shear stress fluctuations is proposed in Eq. (6), which generalizes that from Sumer et al. [40]. It can be used to modify the calculation of sediment transport rate not only for uniform open-channel flow but also for the vortex flow in front of a cylinder. The validation of the new proposed sediment transport model indicates that it improves the accuracy of scour prediction for the initial scouring process in front of a cylinder.

\section{Acknowledgements}

The authors would like to thank the two anonymous reviewers for their constructive comments and suggestions during the revision of the manuscript. We also thank Dr. Yilin Yang for her help on the supplementary experiment. This work was supported by the National Natural Science Foundation of China [grant number 51578062] and the Science and Technology Research and Development Projects of China Railway [grant number 2014G009-H]. 


\section{Appendix A.}

In this Appendix, to examine the convergence of time-averaged results based on the present settings for sampling time and image number, we added a test to analyze the effects of time $(T)$ and image number $\left(n_{i}\right)$ on the time-averaged flow field.

We selected four different parameter settings (i.e., $T=0.1 \mathrm{~s}, n_{i}=50 ; T=1 \mathrm{~s}, n_{i}=500 ; T=1.6 \mathrm{~s}, n_{i}=800$; and $\left.T=2 \mathrm{~s}, n_{i}=1000\right)$ for case C9400 and obtained their respective time-averaged flow field, as listed in the following Fig. 16 from top to bottom. This figure clearly illustrates that the result for $T=0.1 \mathrm{~s}$ and $n_{i}=50$ is obviously different from the other results, e.g. by the presence of three or even four vortices. This discrepancy is due to small number of sampling images $\left(n_{i}\right)$ and short sampling period $(T)$. However, with the increase of $T$ and $n_{i}$, the time-averaged flow fields tend to become more and more consistent, indicating the final convergence of the averaged result. To be specific: 1) from the time-averaged streamline pattern, reasonable agreement is found regarding the vortex typologies for the latter three cases, especially for the cases of $n_{i}=800$ and 1000, and they are almost the same, regardless of the position or size of the vortices; 2) the contours of each time-averaged quantity for the latter three cases remain essentially similar, and the results for both cases with $n_{i}=800$ and 1000 are almost the same, indicating the convergence of the time-averaged results for $T=2 \mathrm{~s}$ and $n_{i}=1000$ case. 


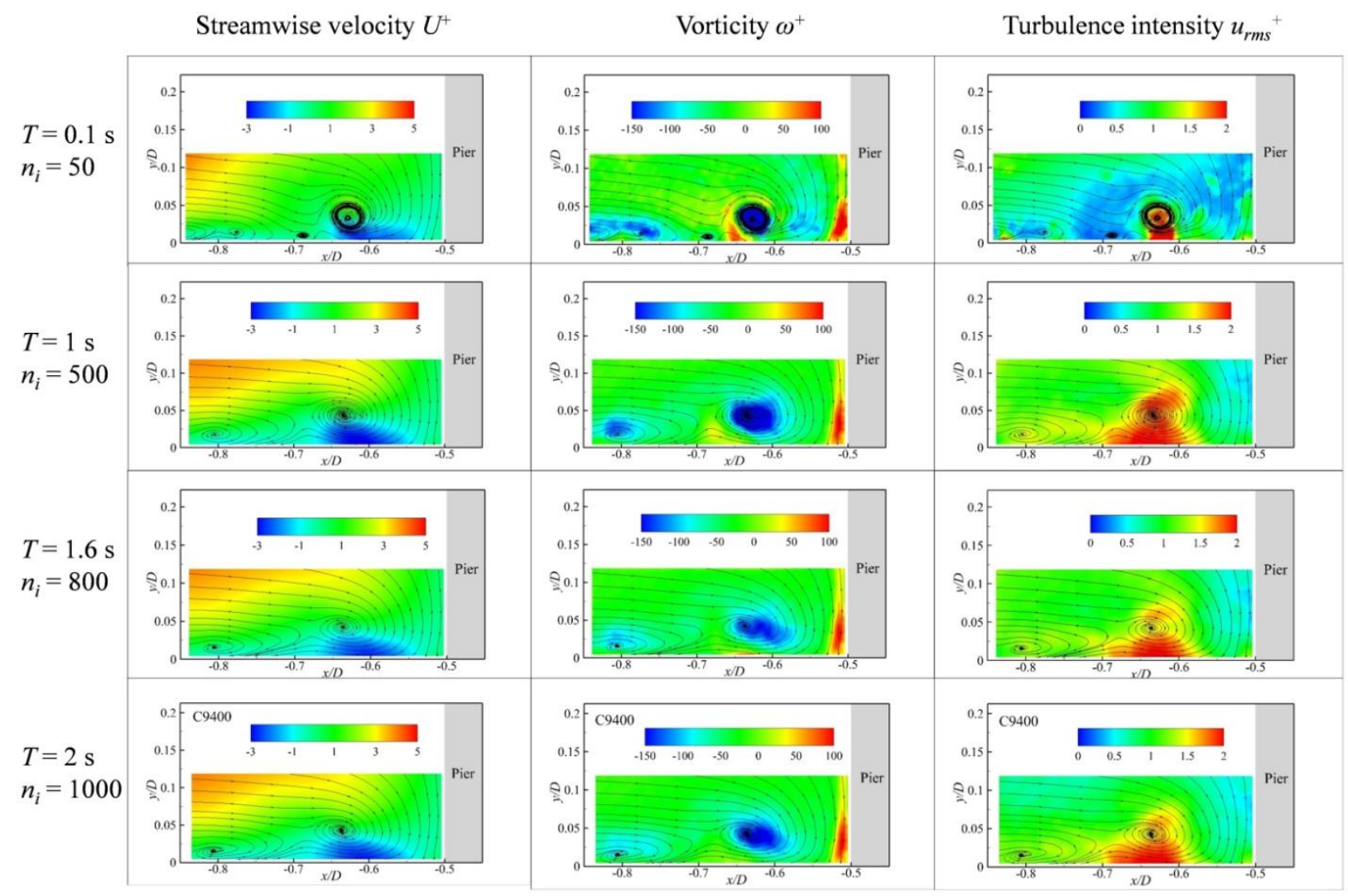

Fig. 16 Effects of sampling time $(T)$ and image number $\left(n_{i}\right)$ on the time-averaged flow field for case C9400.

\section{Appendix B.}

In the present experiment, due to limitations in camera memory, a total of 1000 frames was captured every time, corresponding to a sampling period of $T=2 \mathrm{~s}$. To acquire an increased number of frames, we repeated the measurement for $n$ times corresponding to $n T$ where $n=1 \sim 10$, and thus the maximum number of frames is 10,000 . To reflect the effect of the number of periods $(n)$, we calculated the statistical quantities of bed shear stress for different $n$ for case C9400, as shown in Fig. 17. This figure clearly demonstrates that the profiles almost overlap, especially for the profiles of $n=5$ and $n=10$, implying that the statistical convergence may be achieved. 

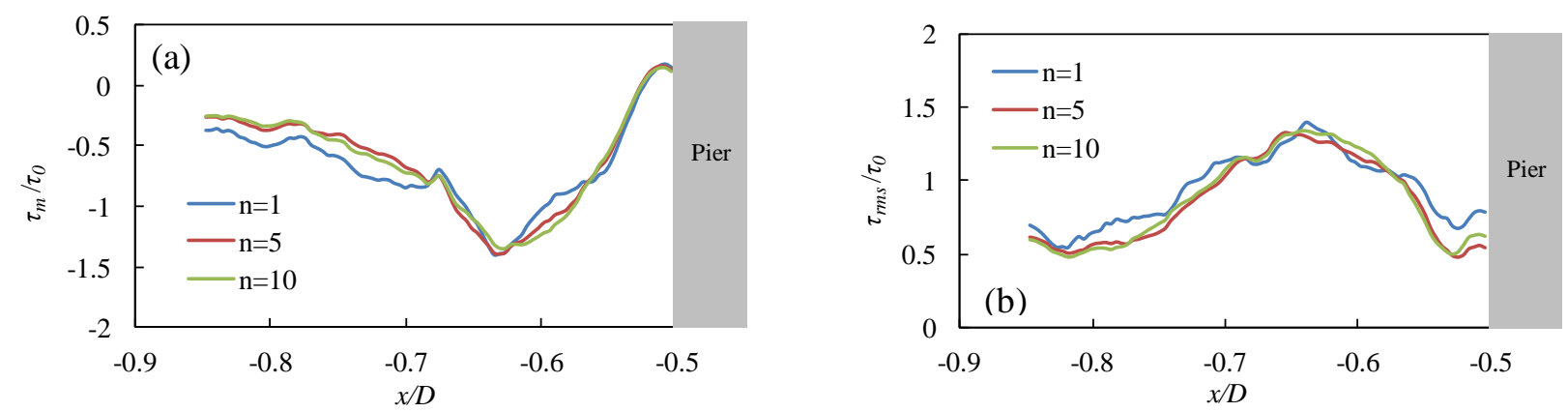

Fig. 17 Statistical error of (a) mean bed shear stress $\tau_{m}$ and (b) RMS of the bed shear stress $\tau_{r m s}$ for different number of sampling period $n$.

To further quantitatively investigate statistical convergence, the error of the bed shear stress statistics for different number of sampling period $(n)$ was calculated, based on the following equation:

$$
\text { Error }=\sqrt{\frac{1}{N} \sum_{i=1}^{N}\left(\frac{R_{i}^{n}-R_{i}^{10}}{R_{i}^{10}}\right)^{2}}
$$

where $N=85$ is the total number of data, $R^{n}$ denotes the statistical quantities of the bed shear stress (e.g., $\tau_{m}$ or $\left.\tau_{r m s}\right)$. Here we recognized the statistical result for a total number of 10 periods $\left(R^{10}\right)$ as a standard. Figure 18 demonstrates the variation of error with the number of period $(n)$. Generally, all the errors are no more than $4 \%$, and with the increase of $n$, the error is significantly reduced, particularly, to smaller than $2 \%$ when $n>4$. For $n>5$, the error is small (approximately $<1 \%$ ) indicating reasonable accuracy can seemingly be achieved.
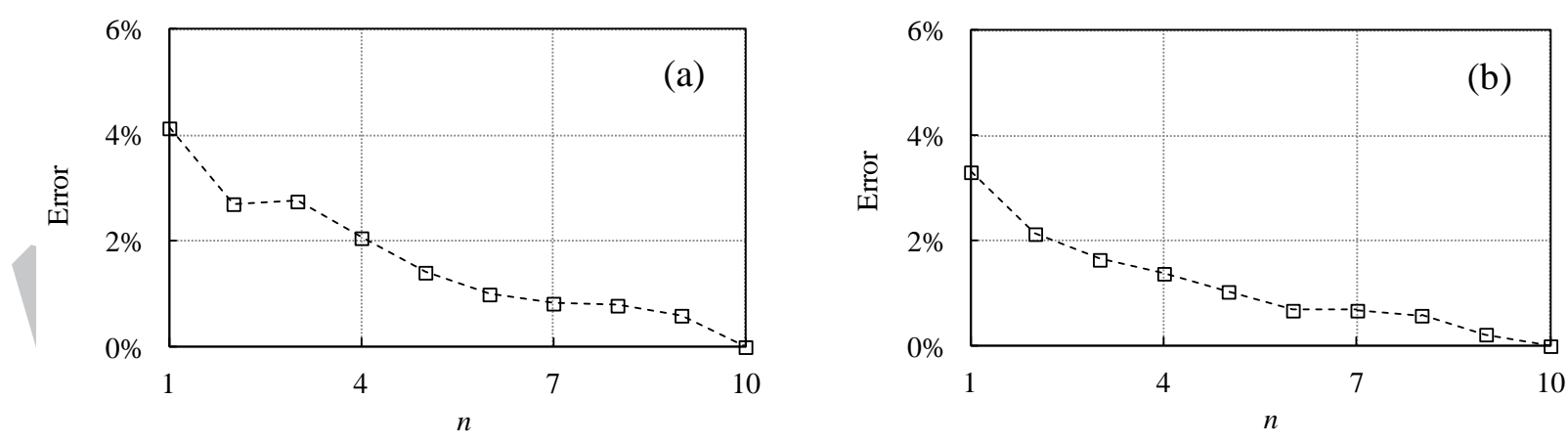

Fig. 18 Statistical error of (a) mean bed shear stress and (b) RMS of the bed shear stress versus number of period $n$. 


\section{References}

[1] Melville, B. W., \& Coleman, S. E. (2000). Bridge scour. Colorado: Water Resources Publications.

[2] Qi, M. L., Li, J. Z., \& Chen, Q. G. (2016). Comparison of existing equations for local scour at bridge piers: $\begin{array}{llll}\text { parameter } \quad \text { influence } & \text { and } \quad \text { validation. Natural } & \text { Hazards, 82(3), }\end{array}$ https://doi.org/10.1007/s11069-016-2287-z

[3] Dargahi, B. (1990). Controlling mechanism of local scouring. Journal of Hydraulic Engineering, 116(10), 1197-1214. https://doi.org/10.1061/(ASCE)0733-9429(1990)116:10(1197)

[4] Sumer, B. M., \& Fredsøe, J. (2002). The Mechanics of Scour in the Marine Environment. World Scientific, Singapore. pp. 536.

[5] Graf, W. H., \& Yulistiyanto, B. (1998). Experiments on flow around a cylinder; the velocity and vorticity fields. Journal of Hydraulic Research, 36(4), 637-653. https://doi.org/10.1080/00221689809498613

[6] Muzzammil, M., \& Gangadhariah, T. (2003). The mean characteristics of horseshoe vortex at a cylindrical pier. Journal of Hydraulic Research, 41(3), 285-297. http://dx.doi.org/10.1080/00221680509500157

[7] Dey, S., \& Raikar, R. V. (2007). Characteristics of horseshoe vortex in developing scour holes at piers, Journal of Hydrauic Engineering, 133(4), 399-413. http://dx.doi.org/10.1061/(ASCE)0733-9429(2007)133:4(399)

[8] Ozturk, N. A., Akkoca, A., \& Sahin, B. (2008). Flow details of a circular cylinder mounted on a flat plate. Journal of Hydraulic Research, 46(3), 344-355. http://dx.doi.org/10.3826/jhr.2008.3126

[9] Lin, C., Chiu, P. H., \& Shieh, S. J. (2002). Characteristics of horseshoe vortex system near a vertical plate-base plate juncture. Experimental Thermal and Fluid Science, 27, 25-46.

[10] Huang, R. F., Hsu, C. M., \& Lin, W. C. (2014). Flow characteristics around juncture of a circular cylinder mounted normal to a flat plate. Experimental Thermal and Fluid Science, 55, 187-199. http://dx.doi.org/10.1016/j.expthermflusci.2014.03.012

[11] Huang, R. F., Hsu, C. M., \& Cheng, T. H. (2018). Effects of upstream tetrahedron length on flow characteristics around juncture of circular cylinder and flat plate. Experimental Thermal and Fluid Science, 92, $295-305$. https://doi.org/10.1016/j.expthermflusci.2017.12.006

[12] Apsilidis, N., Diplas, P., Dancey, C. L., \& Bouratsis, P. (2015). Time-resolved flow dynamics and Reynolds number effects at a wall-cylinder junction. Journal of Fluid Mechanics, 776, $475-511$. https://doi.org/10.1017/jfm.2015.341

[13] Apsilidis, N., Diplas, P., Dancey, C. L., \& Bouratsis, P. (2016). Effects of wall roughness on turbulent junction 
flow characteristics. Experiments in Fluids, 57(1), 1-16. https://doi.org/10.1007/s00348-015-2098-0

[14] Chen, Q. G., Qi, M. L., Zhong, Q., \& Wang, X. K. (2017). Experimental study on the multimodal dynamics of the turbulent horseshoe vortex system around a circular cylinder, Physics of Fluids, 29, 015106. http://dx.doi.org/10.1063/1.4974523

[15] Kirkil, G., Constantinescu, G., \& Ettema, R. (2005). The horseshoe vortex system around a circular bridge pier on a flat bed. Impacts on Global Climate Change, 1-12. https://doi.org/10.1061/40792(173)414

[16] Kirkil, G., \& Constantinescu, G. (2015). Effects of cylinder Reynolds number on the turbulent horseshoe vortex system and near wake of a surface-mounted circular cylinder. Physics of Fluids, 27, 075102. https://doi.org/10.1063/1.4923063

[17] Schanderl, W., Jenssen, U., Strobl, C., Manhart, M., \& Schanderl, W. (2017). The structure and budget of turbulent kinetic energy in front of a wall-mounted cylinder. Journal of Fluid Mechanics, 827, $285-321$. https://doi.org/10.1017/jfm.2017.486

[18] Escauriaza, C., \& Sotiropoulos, F. (2011a). Reynolds number effects on the coherent dynamics of the turbulent horseshoe vortex system. Flow, Turbulence and Combustion, 86, 231-262. https://doi.org/10.1007/s10494-010-9315-y

[19] Escauriaza, C., \& Sotiropoulos, F. (2011b). Lagrangian model of bed-load transport in turbulent junction flows. Journal of Fluid Mechanics, 666(1), 36-76. https://doi.org/10.1017/S0022112010004192

[20] Link, O., González, C., Maldonado, M., \& Escauriaza, C. (2012). Coherent structure dynamics and sediment particle motion around a cylindrical pier in developing scour holes. Acta Geophysica, 60(6), 1689-1719. https://doi.org/10.2478/s11600-012-0068-y

[21] Roulund, A., Sumer, B. M., Fredsøe, J., \& Michelsen, J. (2005). Numerical and experimental investigation of flow and scour around a circular pile. Journal of Fluid Mechanics, 534, 351-401. https://doi.org/10.1017/S0022112005004507

[22] Nagata, N., Hosoda, T., Nakato, T., \& Muramoto, Y. (2005). Three-dimensional numerical model for flow and bed deformation around river hydraulic structures. Journal of Hydraulic Engineering, 131(12), $1074-1087$. http://dx.doi.org/10.1061/(ASCE)0733-9429(2005)131:12(1074)

[23] Liu, X. F., \& García, M. H. (2008). Three-dimensional numerical model with free water surface and mesh deformation for local sediment scour. Journal of Waterway Port Coastal \& Ocean Engineering, 134(4), $203-217$. https://doi.org/ 10.1061/(ASCE)0733-950X(2008)134:4(203)

[24] Zhao, M., Cheng, L., \& Zang, Z. (2010). Experimental and numerical investigation of local scour around a 
submerged vertical circular cylinder in steady currents. Coastal Engineering, 57(8), $709-721$. https://doi.org/10.1016/j.coastaleng.2010.03.002

[25] Abbasnia, A. H., \& Ghiassi, R. (2011). Improvements on bed-shear stress formulation for pier scour computation. International Journal for Numerical Methods in Fluids, 67, $383-402$. https://doi.org/10.1002/fld.2372

[26] Stahlmann, A. (2014). Numerical and experimental modeling of scour at foundation structures for offshore wind turbines. Journal of Ocean and Wind Energy, 1(2), 82-89.

[27] Afzal, M. S., Bihs, H., Kamath, A., \& Arntsen, Ø. A. (2015). Three-dimensional numerical modeling of pier scour under current and waves using level-set method. Journal of Offshore Mechanics and Arctic Engineering, 137, 032001. https://doi.org/032001. 10.1115/1.4029999

[28] Baykal, C., Sumer, B. M., Fuhrman, D. R., Jacobsen, N. G., \& Fredsøe, J. (2015). Numerical investigation of flow and scour around a vertical circular cylinder. Philosophical Transactions, 373, 20140104. https://doi.org/10.1098/rsta.2014.0104

[29] Baykal, C., Sumer, B. M., Fuhrman, D. R., Jacobsen, N. G., \& Fredsøe, J. (2017). Numerical simulation of scour and backfilling processes around a circular pile in waves. Coastal Engineering, 122, 87-107. https://doi.org/10.1016/j.coastaleng.2017.01.004

[30] Xiong, W., Cai, C. S., Kong, B., \& Kong, X. (2016). CFD simulations and analyses for bridge-scour development using a dynamic-mesh updating technique. Journal of Computing in Civil Engineering, 30(1), 04014121. https://doi.org/10.1061/(ASCE)CP.1943-5487.0000458

[31] Khosronejad, A., Kang, S., \& Sotiropoulos, F. (2012). Experimental and computational investigation of local scour around bridge piers. Advances in Water Resources, 37, 73-85. https://doi.org/10.1016/j.advwatres.2011.09.013

[32] Chang, W. Y., Constantinescu, G., Tsai, W. F., \& Lien, H. C. (2011). Coherent structure dynamics and sediment erosion mechanisms around an in-stream rectangular cylinder at low and moderate angles of attack. Water Resources Research, 47, W12532. https://doi.org/10.1029/2011WR010586

[33] Kim, H. S., Nabi, M., Kimura, I., \& Shimizu, Y. (2014). Numerical investigation of local scour at two adjacent cylinders. Advances in Water Resources, 70, 131-147. https://doi.org/10.1016/j.advwatres.2014.04.018

[34] Kim, H. S., Nabi, M., Kimura, I., \& Shimizu, Y. (2015). Computational modeling of flow and morphodynamics through rigid-emergent vegetation. Advances in Water Resources, 84, 64-86. https://doi.org/10.1016/j.advwatres.2015.07.020

[35] Stoesser, T. (2014). Large-eddy simulation in hydraulics: Quo Vadis?. Journal of Hydraulic Research, 52(4), 
441-452. https://doi.org/10.1080/00221686.2014.944227

[36] Dargahi, B. (1989). The turbulent flow field around a circular cylinder. Experiments in Fluids, 8, 1-12. https://doi.org/10.1007/BF00203058

[37] Schanderl, W., \& Manhart, M. (2016). Reliability of wall shear stress estimations of the flow around a wall-mounted cylinder. Computers and Fluids, 128, 16-29. https://doi.org/10.1016/j.compfluid.2016.01.002

[38] Cheng, N. S., Sumer, B. M., \& Fredsøe, J. (2003). Investigation of bed shear stresses subject to external turbulence. International Journal of Heat and Fluid Flow, 24, 816-824. https://doi.org/10.1016/S0142-727X(03)00088-2

[39] Cheng, N. S., Tang, H. W., \& Zhu, L. J. (2004). Evaluation of bed load transport subject to high shear stress fluctuations. Water Resources Research, 40, W05601. https://doi.org/10.1029/2003WR003001

[40] Sumer, B. M., Chua, L. H. C., Cheng, N. S., \& Fredsøe, J. (2003). Influence of turbulence on bed load sediment transport. Journal of Hydraulic $\quad$ Engineering, 129(8), 585-596. http://dx.doi.org/10.1061/(ASCE)0733-9429(2003)129:8(585)

[41] Unger, J., \& Hager, W. H. (2007). Down-flow and horseshoe vortex characteristics of sediment embedded bridge piers. Experiments in Fluids, 42, 1-19. https://doi.org/10.1007/s00348-006-0209-7

[42] Beheshti, A. A., \& Ataie-Ashtiani, B. (2016). Scour hole influence on turbulent flow field around complex bridge piers. Flow, Turbulence and Combustion, 97(2), 451-474. https://doi.org/10.1007/s10494-016-9707-8

[43] Scarano, F. (2002). Iterative image deformation methods in PIV. Measurement Science and Technology, 13, R1-R19.

[44] Chen, Q. G., Adrian, R. J., Zhong, Q., Li, D. X., \& Wang, X. K. (2014). Experimental study on the role of spanwise vorticity and vortex filaments in the outer region of open-channel flow. Journal of Hydraulic Research, 52(4), 476-489. http://dx.doi.org/10.1080/00221686.2014.919965

[45] Qi, M. L., Li, J. Z., Chen, Q. G., \& Zhang, Q. F. (2018). Roughness effects on near-wall turbulence modelling for open-channel flows. Journal of Hydraulic Research (published online). http://dx.doi.org/10.1080/00221686.2017.1399931

[46] Justesen, P. (1991). A note on turbulence calculations in the wave boundary layer. Journal of Hydraulic Research, 29(5), 699-711. http://dx.doi.org/10.1080/00221689109498985

[47] Paik, J., Escauriaza, C., \& Sotiropoulos, F. (2007). On the bimodal dynamics of the turbulent horseshoe vortex system in a wing-body junction. Physics of Fluids, 19, 045107. https://doi.org/10.1063/1.2716813

[48] Escauriaza, C., \& Sotiropoulos, F. (2011c). Initial stages of erosion and bed form development in a turbulent flow 
around a cylindrical pier. Journal of Geophysical Research, 116, F03007. https://doi.org/10.1029/2010JF001749

[49] Graf, W. H., \& Istiarto. I. (2002). Flow pattern in the scour hole around a cylinder. Journal of Hydraulic Research, 40(1), 13-20. http://dx.doi.org/10.1080/00221680209499869

[50] Van Rijn, L. C. (1984). Sediment transport, part I: Bed load transport, Journal of Hydraulic Engineering, 110(10), 1431-1456. http://dx.doi.org/10.1061/(ASCE)0733-9429(1984)110:10(1431)

[51] Guo, J. (2014). Semi-analytical model for temporal clear-water scour at prototype piers. Journal of Hydraulic Research, 52(3), 366-374. http://dx.doi.org/10.1080/00221686.2013.877527 
Highlights

- $\quad$ The THV-induced fluctuations of bed shear stress are comparable to those of mean.

- The PDF of instantaneous bed shear stress exhibits a double-peaked distribution.

- Sediment transport rate based on mean bed shear stress is underestimated

- A relationship between sediment transport and THV-induced fluctuations is established. 